\title{
Savoirs paysans autour des huiles d'olive, (zaytun, Olea europaea var. europaea) et d'oléastre, (əl-bərri, Olea europaea var. sylvestris) Rif, nord du Maroc
}

De la reproduction des arbres aux pratiques alimentaires

Farmer's knowledge around olive (zaytun, Olea europaea var. europaea)) and oleaster (əl-bərri, Olea europaea var. sylvestris) oils, Rif, Northern Morocco:

From tree reproduction to food practices

Yildiz Aumeeruddy-Thomas et Dominique Caubet

\section{OpenEdition}

Édition électronique

URL : https://journals.openedition.org/ethnoecologie/3198

DOI : $10.4000 /$ ethnoecologie.3198

ISSN : 2267-2419

Éditeur

Laboratoire Éco-anthropologie

Référence électronique

Yildiz Aumeeruddy-Thomas et Dominique Caubet, « Savoirs paysans autour des huiles d'olive, (zaytun, Olea europaea var. europaea) et d'oléastre, (əl-bərri, Olea europaea var. sylvestris) Rif, nord du Maroc », Revue d'ethnoécologie [En ligne], Supplément 1 | 2017, mis en ligne le 17 octobre 2017, consulté le 19 novembre 2021. URL : http://journals.openedition.org/ethnoecologie/3198 ; DOI : https://doi.org/ 10.4000/ethnoecologie.3198

Ce document a été généré automatiquement le 19 novembre 2021.

Revue d'ethnoécologie est mis à disposition selon les termes de la licence Creative Commons Attribution - Pas d'Utilisation Commerciale - Pas de Modification 4.0 International. 


\section{Savoirs paysans autour des huiles d'olive, (zaytun, Olea europaea var. europaea) et d'oléastre, (əl-bərri, Olea europaea var. sylvestris) Rif, nord du Maroc}

De la reproduction des arbres aux pratiques alimentaires

Farmer's knowledge around olive (zaytun, Olea europaea var. europaea)) and oleaster (əl-bərri, Olea europaea var. sylvestris) oils, Rif, Northern Morocco: From tree reproduction to food practices

Yildiz Aumeeruddy-Thomas et Dominique Caubet

\section{Introduction}

Dans ce papier, nous nous intéressons centralement aux produits de l'olivier et de l'oléastre du nord du Maroc, aux modalités techniques d'obtention des olives de table et des différents types d'huile et ce que ces produits représentent pour les habitants. Il s'agit de comprendre si le Rif détient une particularité sur ce sujet et comment l'huile (ou les huiles) participent à la vie sociale des habitants du Rif autour de la table, dans les moulins et sur les marchés. Nous discutons également les échanges au sein des groupes arabophones du Rif à propos de ces savoirs en les situant dans le cadre d'échanges plus vastes au sein des souks et matrimoniaux. Nous nous intéressons également à la façon dont la perception des types d'oléastres classés selon l'huile que contiennent leurs fruits a pu influencer des processus pré-domesticatoires et de création variétale au cours de l'histoire.

2 En outre, ce que nous apporte la linguistique, soulève plusieurs questions importantes quant au rôle joué par les anciennes populations berbères dans le développement de 
l'oléiculture à l'échelle méditerranéenne. Selon Chaker (2013) «le berbère possède deux dénominations fondamentales de l'olivier-oléastre: azəmmur, "olivier » ou "oléastre" selon les régions et āliw/ālew, limitée au touareg et qui désigne spécifiquement une variété saharienne d'olivier sauvage. Au fil de son analyse des termes berbères et s'appuyant sur des idées aussi émises par Meillet (1975 : 302-303), Chaker (2013) considère que āliw, ālew / /āliwən, bien qu'utilisé uniquement en Touareg de nos jours pour désigner l'olivier Saharien (O. europaea subsp. Laperrinei) qui pousse exclusivement dans les régions arides et semi arides du sud, correspondrait à un terme équivalent ou proche en Kabylie, pour un arbre de la famille des Oléacées, le filaire (Phillyrea sp.) qui ressemble beaucoup à l'olivier. Le filaire est nommé en berbère kabyle wala(w) ou tamət wala. Chaker émet ainsi l'hypothèse sur le plan linguistique que wala pourrait être l'équivalent kabyle de āliw. S'appuyant en outre sur les travaux de Camps (1985, cité par Chaker 2013) sur la période cyrénaïque durant laquelle des contacts étaient déjà établis entre Grecs et Berbères, Chaker (ibid.) propose l'idée plausible à ses yeux que les Berbères auraient d'abord nommé l'olivier, terme ensuite transmis aux Grecs au VII siècle avant J.-C, avant sa première utilisation par les Grecs au mycéen (xvI siècle avant J.-C.), puis olea par les romains. En outre G. Camps (selon Chaker) «a consacré un long passage à l'olivier dans son Massinissa (1961: 87-91) et considère que les Berbères pratiquaient la greffe de l'oléastre avant l'influence phénicienne et signale [...] que "les habitants de Djerba savaient tirer de l'huile des fruits de l'oléastre ». Autrement dit, les Berbères exploitaient déjà l'olivier au milieu du IV siècle avant J.-C c'est à dire il y a environ 2500 ans, et pratiquaient vraisemblablement aussi la greffe. Cette synthèse linguistique et historique sur les façons de nommer l'olivier au Maghreb ouvre de nouvelles perspectives concernant les liens aux usages de l'huile d'oléastre ainsi que des techniques de reproduction par la greffe apportent de «l'huile» à notre moulin comme nous le verrons plus loin!

3 Enfin, les vergers traditionnels du pays Jbala au nord du Maroc, font face désormais à une avancée rapide des vergers industriels d'oliviers fondée sur la reproduction des arbres par des boutures élevées dans des pépinières telles que celles situées à Marrakech ou Meknès avec un mode de développement de ces vergers soutenu par le Plan Maroc Vert, plan de développement agricole en cours au Maroc. Ces développements destinés à augmenter l'huile d'olive pour l'exportation, pourraient mettre en péril les vergers traditionnels et la grande diversité des huiles locales, ainsi qu'un maillon manquant de la domestication de l'olivier que nous avons mis en lumière dans une étude alliant approches ethnobotanique, génétique et géographique (Aumeeruddy-Thomas et al. 2016 et 2017). La question que nous discutée en conclusion est : quel pourrait être le rôle des chercheurs en collaboration avec les collectivités locales pour une patrimonialisation de certains pans de ces agroécosystèmes et des savoirs et savoir-faire paysans associés.

4 L'ensemble des travaux présentés dans ce papier cherche à situer la place des huiles, notamment des huiles d'olive et d'oléastre dans un cadre général de réflexion sur le Rif. Il s'agit en effet de préciser le rôle des oliviers et des chaînes opératoires de plantation, de récolte et de production d'huile dans la relation des Jbala à leur territoire et aux autres groupes arabophones du Rif, dans leur mode de vie et leurs rapports à autrui, humains et non-humains. Notons que nous n'avons pas travaillé dans des zones strictement berbérophones du Rif sur ce sujet. 


\section{Sites d'étude et méthodes ${ }^{1}$}

5 Nous présentons tout d'abord les éléments clés de la bibliographie sur le sujet traité afin de situer l'olivier et les usages des huiles dans un contexte historique et géographique avant toute chose pour affiner notre compréhension des pratiques autour de l'olivier et de l'oléastre.

6 Nos données empiriques sont fondées sur deux missions conjointes ethnobotanique et linguistique effectuées en février 2014 à Msek chez les Bni Itteft, une fraction des Ait Aissa, dans le Rif (occidental) dans une communauté arabophone vivant à la frontière de la berbérophonie (voir Caubet et Aumeeruddy-Thomas ce volume) et en avril-mai 2014 en pays Jbala à Ain Mediouna dans le village de Aouina Melha [litt. La source salée], dans le Pré-Rif (Figure 1). Nous avons également visité leurs voisins Hyayna une confédération de trois tribus d'origine bédouine qui se sont installées au XVI-XVII siècles dans cette région entre deux affluents du fleuve Sebou, l'oued Ouergha au nord et l'Oued Inaouène au sud. Provenant de groupes arabophones du sud, ce sont d'anciens groupes bédouins devenus des agriculteurs sédentaires qui ont été constitués en groupe de soldats dit «Guich» et furent installés dans la région de Fès par les Saâdiens. Ces soldats obtenaient des terres dites "Guich» par le Makhzen (El Moubaraki 1989, Szymanski 1970 et carte en annexe). Le village Hyayna visité est Swetiyyin situé au nord est de Tissa (Figure 1). Les dialectes pré-hilaliens des Jbala se sont constitués à partir de la rencontre entre des migrations arabes arrivées au nord du Maroc au viI ${ }^{e}$ siècle, rencontrant les sociétés berbères Senhaja du Rif et influencées également par l'arabe andalou. Les Hyayna pour leur part ont retenu des caractéristiques du parler bédouin très clairement identifiées par les habitants de Ain Mediouna par exemple comme distinctes de leur propre parler et que nous avons identifiées sur place. Ils ont été influencés également de diverses façons, bien que plus tardivement, par les Berbères Senhaja déjà arabisés qui les ont accueillis (El Moubaraki 1989).

7 Diverses autres missions menées quasi annuellement par Yildiz Aumeeruddy-Thomas chez les Bni Ahmed, à Cherafate, à Chefchaouen dans le Rif central et dans le Pré-Rif à Ouezzane, Ain Dorij, Masmouda, Sidi Redouane depuis 2006, ont permis également de recueillir divers corpus en plus de ceux présentés ici, dont nous n'avons retenu que des éléments (termes et absence ou présence de certaines pratiques) à titre de comparaison.

8 Nous avons été reçu à Ain Mediouna par Fouzia el Ghazaz, alors en thèse de linguistique à l'Université Sidi Ben Abdellah à Fès et dont c'est le village d'origine. À Msek, nous étions en compagnie d'Amal Maghdad et Khalid El Jettari. Amal Maghdad, qui est traductrice à Tanger et originaire de la région de Msek, nous a servi d'interprète. Elle avait elle-même effectué un mémoire de licence d'espagnol sous la direction de Simon Lévy dans cette région auparavant (Maghdad 1993). El Jettari était alors en master à l'Université d'Oujda, travaillant sur les dialectes berbères des Bni Itteft (El Jettari 2017). 
Figure 1 : Sites visités entre 2006 et 2015 (points rouge sur la carte), Rif, nord du Maroc

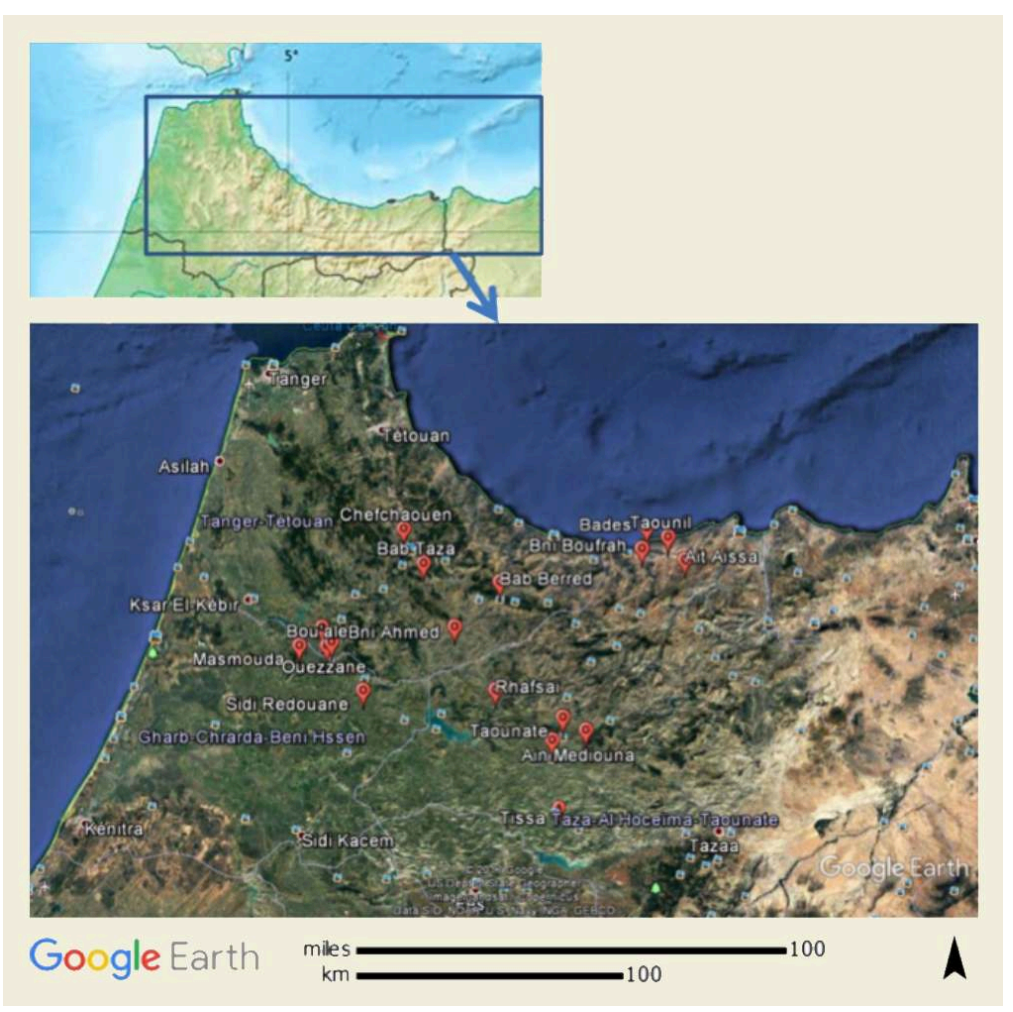

\section{Résultats}

\section{De la reproduction des arbres à la production des huiles}

\section{Vergers-forêts d'oliviers du Rif : une spécificité à forte valeur patrimoniale}

L'olivier a vraisemblablement été domestiqué dans l'est méditerranéen et divers travaux menés par des archéobotanistes et des généticiens montrent que les oliviers domestiqués suivent une route de diffusion méditerranéenne est-ouest (Kaniewsky et al. 2012, Besnard et al. 2013, Diez et al. 2015). D'autres travaux en archéobotanique indiquent cependant que des domestications multi-locales ont aussi eu lieu dans l'ouest méditerranéen il y a environ 5000 ans (Terral \& Arnold-Simard 1996) et que, non seulement les populations d'oliviers augmentent à la faveur de la mise en place de l'agriculture par les populations ouest méditerranéennes, mais que la nature des noyaux et du bois d'olivier change. Ceci a été interprété comme des débuts de processus de sélection. Au Maroc, Ballouche \& Marinval (2003) observent également une présence significative de l'olivier qui est concordante avec le développement de la céréaliculture il y a environ 6000 ans, durant le Néolithique.

Des travaux mêlant ethnobotanique et génétique menés au nord du Maroc depuis 2006, montrent que les anciens vergers traditionnels d'oliviers (Olea europaea var. europaea) greffés sur l'olivier sauvage ou oleastre (Olea europaea var. sylvestris) de la région d'Ouezzane en pays Jbala, constituent un élément clé pour comprendre des processus anciens de domestication de l'olivier vraisemblablement encore favorisés dans ces vergers (Aumeeruddy-Thomas et al. 2017). Ces vergers d'oliviers greffés sur oléastres étaient répandus en Corse, Sardaigne ainsi que dans des petites îles grecques mais, ils 
ont en grande partie disparu. Des vergers de ce type sont présents en petite Kabylie (Brun 1986). Cette technique fort ancienne existe à l'état de trace désormais en Espagne et au Levant et elle a attiré l'attention récemment des généticiens (Diez et al. 2011, Barazani et al. 2014). Ces derniers suggèrent que cette méthode a pu être une des anciennes techniques de domestication de l'olivier et serait passée quelque peu inaperçue. Le Rif est dès lors un des derniers lieux en Méditerranée où ces grands vergers-forêts (car la base des arbres est d'origine forestière) méditerranéens existent, bien que menacés aujourd'hui (Aumeeruddy-Thomas et al. 2016 et 2017).

11 Tout semble indiquer que dès la période grecque antique, une intensification de la culture de l'olivier se met en place sur la rive nord avec des oliviers poussant en rangées dans les champs plutôt qu'en bordure de champs (Chandezon 2007), intensification qui se poursuivra à grande échelle avec les Romains. Les pieds d'oliviers étaient vraisemblablement élevés en pépinières puis transplantés dans les champs, pratique que l'on retrouve chez les Romains avec divers témoignages des agronomes de l'Antiquité (Chandezon ibid.). Ceci s'oppose aux peuplements aléatoires des anciens vergers, ou aux alignements d'oliviers situés en bordures de champs du Rif (Aumeeruddy-Thomas et al. 2017). Nous avons démontré une origine forestière des oléastres greffés dans le Rif, et/ou des recolonisations spontanées par graines. La greffe sur oléastre, valorisée par les agriculteurs en pays Jbala ainsi qu'une présence substantielle d'oléastres non greffés dans ces vergers, favorisent des sélections par les paysans d'oléastres poussant spontanément pour créer des variétés d'olivier perpétuées par greffage. Ceci constitue une des clés de compréhension de l'évolution des oliviers sous domestication et des modalités d'interactions concrètes et symboliques entre les habitants, les arbres sauvages et les variétés cultivées. Cette situation nous permet de repenser l'histoire de la domestication de l'olivier. En effet, cette situation contemporaine permet de cerner les relations entre les hommes et les oliviers, et de mettre en lumière une gamme d'actions humaines et de rôles des oliviers et oléastres parmi lesquelles, les processus d'appropriation des terres agricoles et le rôle clé de l'olivier comme acteur de co-construction des territoires, son rôle comme agent, médiateur de la bāraka divine et des Saints, l'importance des huiles d'oléastres médicinales ainsi que la grande diversité de types d'huiles d'olive pour l'alimentation que nous développons dans ce papier.

Notons qu'au Maroc, les anciens vergers de la Menara et de l'Agdal de Marrakech datant $\mathrm{du} \mathrm{XI} \mathrm{X}^{\mathrm{e}} \mathrm{XII}$ siècles sont caractéristiques des relations arabo-andalouse et furent créés sous le règne de l'Almohade Abdelmoumen (El Faiz \& Bendaoud 2000, Charafi et al. 2008, Khadari \& Moukhli 2016). Un jardin quasi identique existe notamment à Séville et utilise la méthode de bouturage des oliviers. À la Menara, les oliviers sont parfaitement alignés et il existe un système d'irrigation par des xatțāra (canaux d'irrigation développés par les Almoravides dont certaines parties sont souterraines) suggérant une influence des Almoravides et une continuité dans le temps de leur fonctionnement (El Faiz communication personnelle, 2008). Une technique courante dans tout le sud du Maroc que nous avons observée dans la zone de l'arganeraie, à Imintlit dans l'arrièrepays d'Essaouira, consiste à éclater une partie de la base des arbres pour récupérer un rejet. Ceci témoigne du fait que l'arbre était initialement bouturé ce qui permet une reproduction à l'identique par rejets de souche (Aumeeruddy-Thomas \& Simenel, données non publiées). Cette technique évoquée par Zohary et al. (2012) comme possible première forme de multiplication végétative de l'olivier au début de la domestication de l'olivier, diffère de la technique de greffage sur oliviers rencontrée au 
nord de la Tunisie par les Romains, comme montré par les historiens concernant la transcription de l'inscription de Henchir Mettich trouvée sur un site romain en Tunisie (Toutain 1902, Frank 1926) vraisemblablement gravée en 117 après J.-C. À noter que le terme olivatio faisant référence aux taxes prélevées sur les olives apparaitrait selon Toutain (1902) pour la première fois dans les inscriptions romaines sur ce site. La division par rejet de souche (séparation d'un rejet en récupérant une partie de la souche de l'arbre) était déjà présente à Sfax dans le sud de la Tunisie pendant la période coloniale française (Guernier 1947). Le bouturage au Maroc est aussi présent partout au sud de Meknès et de la région de Zerhoun et Volubilis et semble correspondre à une méthode indigène d'autant que les Romains n'ont quasiment eu aucune influence au sud. Dans ces régions, les plantations d'oliviers nécessitent des systèmes d'irrigation pour maintenir en place ces arbres bouturés, alors que ce système n'existe pas, ou à l'état de trace au nord du Maroc où il s'agit d'une agriculture non irriguée (būr). Nous insistons ici sur ces techniques de reproduction, car pour les paysans du nord, le greffage sur oléastre donnerait une huile toute autre que des arbres bouturés industriellement. Selon les paysans jbala, c'est la base, à savoir l'oléastre qui confère sa vitalité à la variété. Ces variétés greffées sur oléastre donneraient selon eux une bien meilleure huile dont le goût différerait de celle des arbres bouturés des projets et dont les racines assez fragiles, fasciculées et non pivotantes confèreraient selon eux, un goût terreux à l'huile.

\section{L'usage de l'huile d'olive et d'oléastre : quelques repères de la préhistoire à nos jours}

L'usage des olives et de l'huile d'olive a fait l'objet de très nombreuses publications dans des disciplines extrêmement diverses allant de leur usage dans le Haut Paléolithique il y a environ 19000 ans au Levant (Kislev et al. 1992), pendant la période antique (Amouretti 1986) et jusqu'à nos jours dans des traités d'ethnobotanique contemporaine (voir par exemple Bellakhdar 2006). Les grandes caractéristiques de l'huile d'olive et d'oléastre montrent qu'elle a servi comme huile de lampe y compris jusqu'à récemment par exemple pour l'oléastre dans l'Atlas marocain (Laoust 1983). L'huile d'olive de grignons, ou zit l-fitōr (une huile de moins bonne qualité obtenue à partir des restes de pâtes pressées et de noyaux) est parfois utilisée au Maroc pour le savon noir, devenu aujourd'hui un savon de luxe. L'huile d'olive était aussi connue comme huile de bains et cosmétiques à travers toute la Méditerranée et l'apogée de ces usages se situe pendant la période antique, les Grecs et les Romains ayant usé et abusé de quantités phénoménales d'huiles d'olive y compris dans le cadre d'onguents pour les athlètes. Cette importance de l'huile d'olive en Méditerranée a donné lieu à des développements techniques dès l'âge du bronze. Il est en effet démontré que des découvertes techniques en matière d'extraction s'accompagnent d'une diffusion très large de la culture de l'olivier et d'un commerce très important transméditerranéen dont les traces sont connues dès l'âge du bronze inférieur il y a environ 3000 av. J.-C. (Salavert 2008). Le développement de l'usage de l'huile d'olive est de ce fait fortement associé au fait colonial romain, dont un des objectifs est de mettre en place des domaines pour la production d'huiles d'olive avec un système de taxes sur les récoltes (ex : olivatio) et de divisions des terres en terres arables, ager, de pâtures saltus et forestières, silva, ainsi que l'élaboration de moulins collectifs fondant les prémices de l'industrialisation de la production d'olives en vue de son exportation. 
14 L'histoire plus récente montre également que la mise au point de nouvelles techniques d'extraction plus performantes et diffusant à travers la Méditerranée confortera un commerce grandissant d'huile d'olive en Méditerranée et à l'international (RamonMünoz 2007). L'huile d'olive fait en effet l'objet d'un commerce abondant pendant la période coloniale française et espagnole, et au début de l'industrialisation $d u \mathrm{XVIII}^{\mathrm{e}}$ jusqu'au XIX ${ }^{e}$ siècle, notamment en Angleterre, en France et aux États-Unis ; de grosses quantités d'huile d'olive brute étaient en effet utilisées pour l'industrie (Ramon-Münoz 2007). Selon cet auteur, c'est au XIX $x^{e}$ siècle que la mise au point de techniques d'extraction d'huiles d'olive fines en Provence par les Français, transmise dans un premier temps en Italie, puis en Espagne, transforma littéralement le marché international de l'huile d'olive. Il devient fréquent cependant que les pays de la rive nord importent de grosses quantités d'huiles, par exemple marocaines, pour les raffiner et mélanger à divers degrés avec des huiles de la rive nord pour en faire des huiles fines, d'où le rôle clé désormais joué par l'Union européenne dans l'établissement des normes de qualité d'huiles à l'échelle internationale (Ramon-Münoz 2007).

En 2013, la Diète Méditerranéenne fut inscrite par l'UNESCO comme Patrimoine Immatériel de l'humanité (https://ich.unesco.org/fr/RL/la-dietemediterraneenne-00884) et ce faisant met en avant un des ingrédients de base de cette diète, l'huile d'olive. L'idée très répandue en anthropologie de l'alimentation et qui ne s'applique pas uniquement à la diète méditerranéenne est reprise pour en justifier la dimension immatérielle à savoir que l'alimentation méditerranéenne implique un ensemble de savoir-faire, de connaissances, de symboliques et de traditions y compris la façon de partager la table (Mediterra 2012). Néanmoins, bien que l'unité de cette alimentation soit désormais mondialement reconnue et que l'huile d'olive semble y participer, l'importance de l'huile d'olive au Maghreb reste somme toute très peu explorée en détail.

16 Au nord du Maroc, l'œuvre de Mouliéras (1895) sur l'usage de l'olive tel qu'analysé par Monkachi (1997), souligne les points importants suivants :

L'huile d'olive est essentielle à divers plats à base de légumineuses, fort appréciés des Jbala : « Le plat qui semble avoir rallié tous les habitants des Djebala est le célèbre bisar : une espèce de purée à base de fèves sèches, arrosée d'huile d'olive, saupoudrée de poudre de piment rouge ». Chez les Bni Mesguilda (dont une partie est à Ouezzane et à Zoumi) Mouliéras 1895 : 450 (cité par Monkachi) note les points suivants : « l'olive noire fendillée au couteau est mise dans l'eau pendant un mois. Ensuite on jette cette eau, on coupe des tranches de citrons, on les brasse avec les olives, on verse sur le tout de l'huile pure et un peu d'eau claire, on saupoudre le tout avec du sel et il n'y a rien, assurent les Djebaliens, de supérieur à ce mets divin». Mouliéras 1895 cité par Monkachi (ibid.) note que «l'huile obtenue des noyaux donne une huile âpre infiniment moins bonne que celle issue de la première pression. Les tourteaux desséchés provenant des noyaux servent de combustible et sont préférés au meilleur bois » Monkachi enfin (1997 : 99), recense les types d'huiles identifiés par Mouliéras (1895): Mouliéras a relevé plusieurs sortes d'huile chez les Bni Zeroual : «celle qui a subi un détritage complet sous des meules de pierre; celle produite par foulage au pied, et l'huile dite ezzoûba qui vient d'olives grillées au four et broyées à coup de maillet dans des gac' $a^{3}$ (grands plats de bois). Les olives broyées avant maturité donnent une huile agréable au goût ». 

des terrains proches de deux de nos sites d'étude, à Taounate et à Ouezzane, décrivent les techniques de pressoirs utilisées par les femmes d'une part à l'échelle domestique afin de produire l'huile des prémices ou Ealwāna avec des petits pressoirs à huile à vis latérales mobiles. Ce moulin est comparé par El Alaoui (ibid.) aux moulins des hommes qui fonctionne avec des pressoirs plus grands à vis latérales fixées. La précision des chaînes opératoires décrites apporte à nos travaux. Nos observations apportent de nouvelles données à travers des approches conjointes anthropologiques, ethnobotaniques et linguistiques. Le tableau 1 présenté plus bas reprendra certains éléments apportés par El Alaoui (ibid.) ainsi que tous les termes que nous avons recueillis par soucis de comparaison et de compilation des termes portant sur la production des huiles au nord du Maroc.

\section{Les différents types d'huiles identifiés}

\section{Eəlwāna ou l'huile des prémices}

\section{Chez les Bni Itteft à Msek}

Msek est un village arabophone au sein de la fraction berbérophone Ait Aissa, de la tribu des Bni Itteft eux-mêmes majoritairement arabophones, dans l'est du Rif

Lors de l'enquête menée à Msek, au lieu-dit Safri, nous avons recueilli les propos suivants auprès d'une jeune femme de 35 ans originaire du village, à propos de cəlwāna, une huile préparée à partir d'olive prématurément récoltée, pas encore pleinement mûre environ au début novembre (Corpus 1 : entretiens Y. Aumeeruddy-Thomas et A. Maghdad, transcription et traduction D. Caubet) :

kanžnīw z-zìtūn, kanžibu-h kanḥmīw l-farṛān, kanḥmīw-ah kayttoḥma mozyān, bhāal lli gāādi ttiyyab al-xobz

21 " On ramasse les olives, on les ramène, on met le four à chauffer, jusqu'à ce qu'il soit bien chaud. Comme si tu allais faire cuire du pain ».

22 kanmash-u, mazyān, nžìu z-zītūn, nnaqqīw-ah, wa neaddlu fì-h, nEaddlu fi-h ybāt l-lìla kāmla, yibas, thass bi-h yābas.

23 «On le frotte bien, on met les olives, on les nettoie et on les met dans le four. On les laisse passer toute la nuit jusqu'à ce qu'elles sèchent, que tu les trouves bien séchées ».

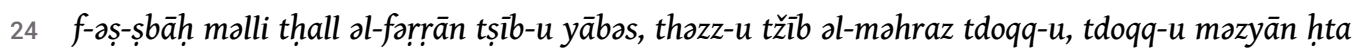
ytdaqq dïk-lo-Ețam dyāl-u

25 «Le lendemain matin, quand tu ouvres le four, tu les sors et tu apportes le mortier et tu les piles, tu les piles bien jusqu'à ce qu'on sépare les noyaux ».

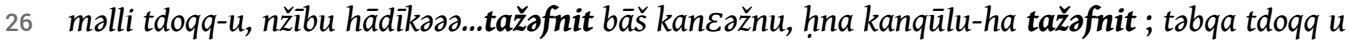

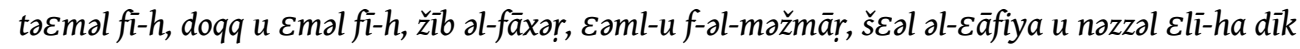

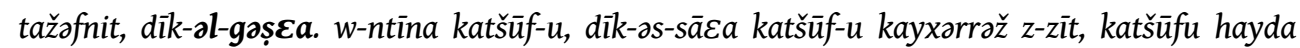
hayda - șāfi u tžìb dorra d al-ḥāyāti u Eassar u hāda șāfi u z-zīt katxrəž məzyān

27 « Une fois qu'ils sont pilés, on apporte cette... tažəfnit, (grand plat) dans lequel on pétrit le pain, nous on l'appelle tažəfnit. Tu continues à piler et à en rajouter, tu apportes le charbon de bois, tu le mets dans le réchaud, tu allumes le feu, et tu y poses la tažznit, la 
gașEa. Et tu surveilles et quand tu vois qu'il y a de l'huile qui sort tu le vois comme ça, alors tu apportes un tissu/derra et tu le presses bien. Et voilà l'huile sort bien »

Cette technique, met en jeu un système de chauffage des olives dans un four, élément important de la vie domestique (cf. Caubet \& Aumeeruddy-Thomas, ce volume), le même four, l-farrāan où l'on fait du pain. L'interlocuteur insiste sur le fait que le chauffage du four est identique à celui du pain, mais il est nettoyé avant que les olives ne soient enfournées. Les olives sont ensuite écrasées au pilon avec une pierre. Le plat dans lequel on malaxe les olives après les avoir pilées et chauffées nommé gasca et qu'elle nomme aussi du nom berbère de tažəfnit est le même type de plat dans lequel elle pétrit aussi le pain. Nous voyons ici ce passage de l'arabe au berbère dans le discours qui témoigne bien de cette situation de frontière évoquée plus en détail dans Caubet \& Aumeeruddy-Thomas (ce volume)

Cette technique nous a intéressées, car elle ne met en jeu de façon totalement inédite aucune forme de presse mécanique, ni de décantation, mais uniquement la transformation des olives dans un four, un concassage à la main pour extraire la pulpe, puis le malaxage à la main avec un peu d'eau et le filtrage de l'huile avec un tissu pressé à la main qu'elle précisera ensuite être un dorra $d$ ol-hāāāti, un foulard de cotonnade fine (Figure 2).

Figure 2 : dərrra d əl-ḥāyāti présenté par Amal Maghdad, Msek, nord du Maroc, Février 2014

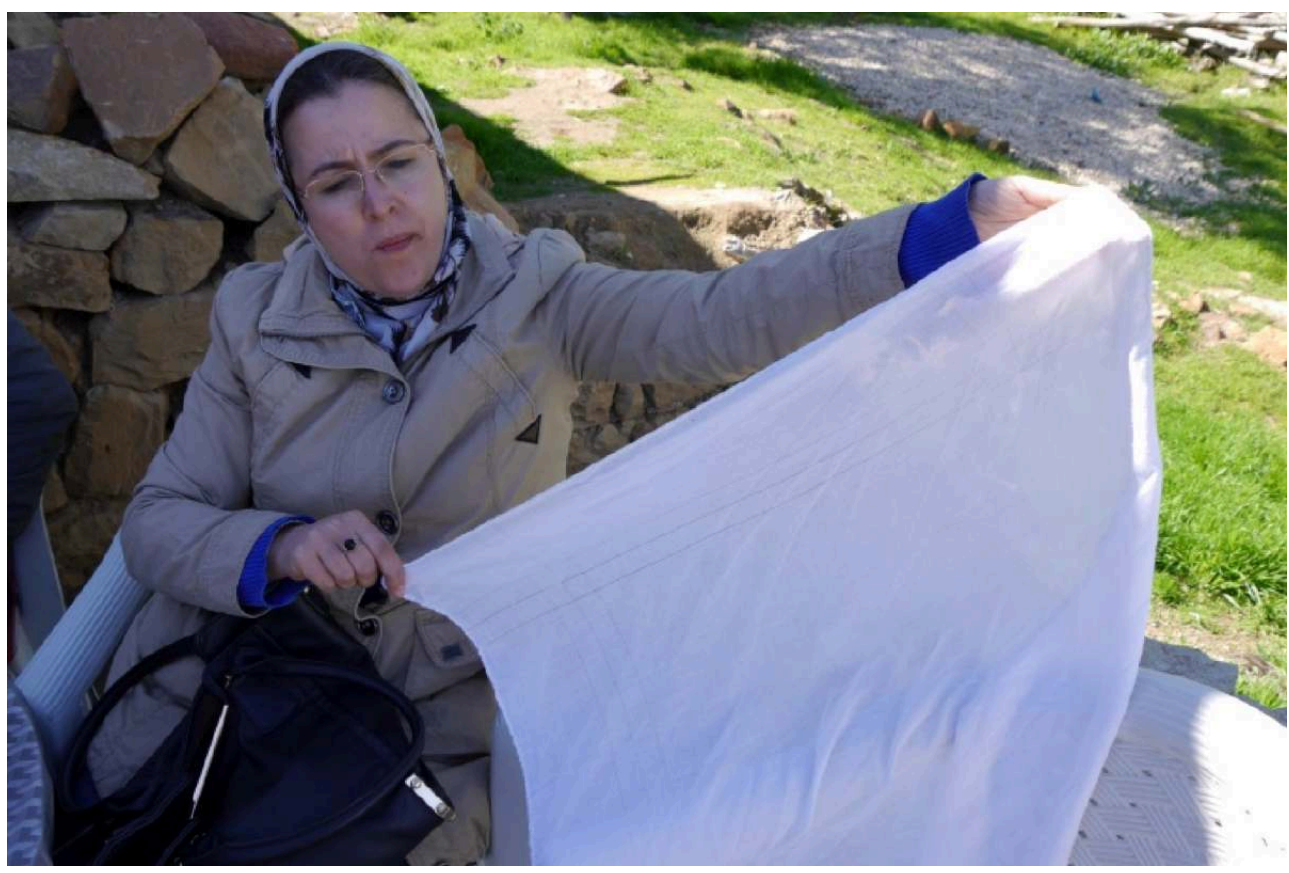

(c) Y. Aumeeruddy-Thomas

Pour hāyāti Mercier (1951) donne les sens suivants: "cotonnade fine, étamine, batiste »; on notera au passage que kās d ol-hāyāti désigne " un verre à thé ordinaire en verre blanc ». Ces tissus au tissage assez lache servent à de multiples usages. Notre hôte nous dit qu'elle extrait l'huile d'amande amère également selon une technique où elle utilise un couscoussier. Il s'agit dès lors de mettre les amandes dans la partie haute du couscoussier; ensuite on met de l'eau dans la partie basse et on chauffe. Les amandes ainsi ramollies sont écrasées au pilon, et l'huile est exprimée et filtrée avec le même tissu. Elle nous dit que l'huile du pistachier térébinthe (zit d-dro) pourrait être fabriquée 
ainsi, mais elle ne le fait pas. Elle sait qu'il s'agit d'une huile médicinale vendue sur les marchés. Cette huile autrefois très utilisée dans le Rif est limitée maintenant à quelques sites (Hmimsa 2009). Quant à l'huile d'amande amère (zit l-lūz marr), elle est utile pour traiter des maux d'oreilles ou très diluée pour le diabète.

Ces résultats mettent en lumière une technique strictement domestique et féminine qui ne met en jeu ni un moulin, ni des techniques de pression mécanique, ni même de décantation. Dans le cadre des approches les plus généralisées d'extraction d'huile d'olive à travers la Méditerranée ces techniques servent habituellement à séparer la pulpe écrasée d'une eau amère connue sous le nom de margines (Brun 1986). Selon El Alaoui (2007), c'est la dessiccation et le chauffage des olives qui permet de se débarrasser de cette amertume. Nous avons ainsi ici une technique possiblement très ancienne, qui permet de désamériser les olives et d'en extraire l'huile avec des moyens très simples. Cette huile nommée Ealwāna par notre hôte est comme nous le verrons, répartie dans toute la zone arabophone du Rif.

Concernant l'utilisation des olives de table, notre hôte fabrique comme beaucoup de familles rifaines, des olives noires simplement macérées dans du sel et gardées anciennement dans des sacs de jute, aujourd'hui remplacés par du plastique tissé pouvant laisser passer le jus amer qui doit sortir de ces olives pour qu'elles puissent être consommées. Elle nous montre son stock d'olives noires qui dit-elle servira toute l'année. Diverses préparations de ces olives permettent d'en améliorer le goût. À Msek, comme partout ailleurs, la générosité des Rifains et des Jbala fait que nous avons été invités à partager une collation dans le cours de l'après-midi où l'huile d'olive, le pain, les olives constituaient les éléments clés de la collation offerte en plus d'un plat de lūbya (haricots blanc), un peu de sel et du cumin.

\section{À Ain Mediouna}

Ain Mediouna se situe dans le Pré-Rif, c'est à dire sur le versant Sud du Rif (Figure 1). Il s'agit d'un groupe social Jbala, parlant un dialecte pré-hilalien tout à fait typique et se disant être des Senhaja. Les voisins des habitants de Ain Mediouna sont les Hyayna, une confédération de tribus située autour de Tissa habitée par des groupes au parler bédouin typique ${ }^{4}$. Les deux groupes reconnaissent les différences de parlers qui les caractérisent l'un et l'autre, ainsi que des styles de vie et des traits culturels distincts. Néanmoins, comme nous le verrons plus bas, les Hyayna et les habitants d'Ain Mediouna échangent des savoirs, des semences, pratiquent des échanges matrimoniaux, à la faveur de rencontres entre les familles dans les souks.

Dans la région d'Ain Mediouna, les éléments recueillis sont donnés dans le deuxième corpus recueilli (Corpus 2 : entretiens ethnobotanique Y-A-Thomas et Fouzia el Ghazaz, Transcription et traduction Fouzia el Ghazaz et D. Caubet)

lla, xəșșu \&a əl-lūn yku zra?

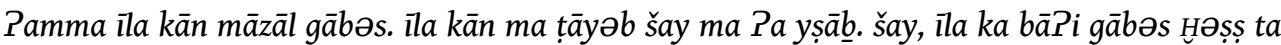
Pa dənzəl Elī-h əš-šta u yərțāb u $\varepsilon \bar{a} d$ Pa nəšwīw-ah

Si elle est encore sèche, Il faut attendre que la pluie tombe pour la ramollir et seulement là nous pouvons la griller. 

partie du séchage des olives dans le four, à la technique rencontrée à Msek dans l'est du Rif. L'enquête précise que les olives doivent être cueillies quand elles sont bleues, ce qui signifie possiblement vert, avant leur maturation. Elles doivent être gorgées par des pluies. L'ensemble de la discussion (non présentée) montre que les femmes cueillent les olives à la main. El Alaoui (2007) de ses travaux à Taounate, dit que les femmes, traient (tasram, hlab ou hallab) les arbres, car il s'agit du même geste que de traire les animaux. Enfin le même type de plat, à fond plat gəșદa, destiné aussi à faire le pain, est utilisé pour le malaxage.

les femmes d'Ain Mediouna utilisent une petite presse mobile meissra qu'elles s'échangent entre les femmes du hameau (lieu-dit Aouina Melha). Elles rajoutent une petite quantité d'eau pour malaxer mais il ne semble pas y avoir de processus de décantation de l'huile obtenue. Il se peut que la faible quantité d'eau ajoutée et le fait que l'huile obtenue soit utilisée immédiatement ne pose pas de problème. Dans l'arganeraie, dans la région de Tiznit et également dans le Souss, comme l'a fait remarquer El Alaoui (2007), et suivant nos propres observations, les femmes rajoutent aussi un peu d'eau pour écraser les noix d'argan légèrement grillées dans un petit moulin. Nous avons pu constater que l'huile d'argan ainsi obtenue peut être vendue dans des bouteilles où il existe un léger fond d'eau. Du sel est mis dans la bouteille, sel qui décante au fond et qui est parfois visible, une pratique qui permet sans doute de s'assurer qu'il n'y ait pas de fermentation.

La meișrra qu'une voisine nous ramène pour nous présenter la technique est en métal, à double vis latérales, une réplique des grandes presses à huile et à vis latérale fixes des moulins. Les olives sont placées dans des petits scourtins (šāmiyya) faits en feuilles de palmier doum ou en jonc, en tout point identiques à ceux utilisés dans des grandes presses des moulins (Figure 3, video 1) 
Figure 3 : La petite presse mobile à عəlwāna,, à Aouina Melha, Rif, nord du Maroc

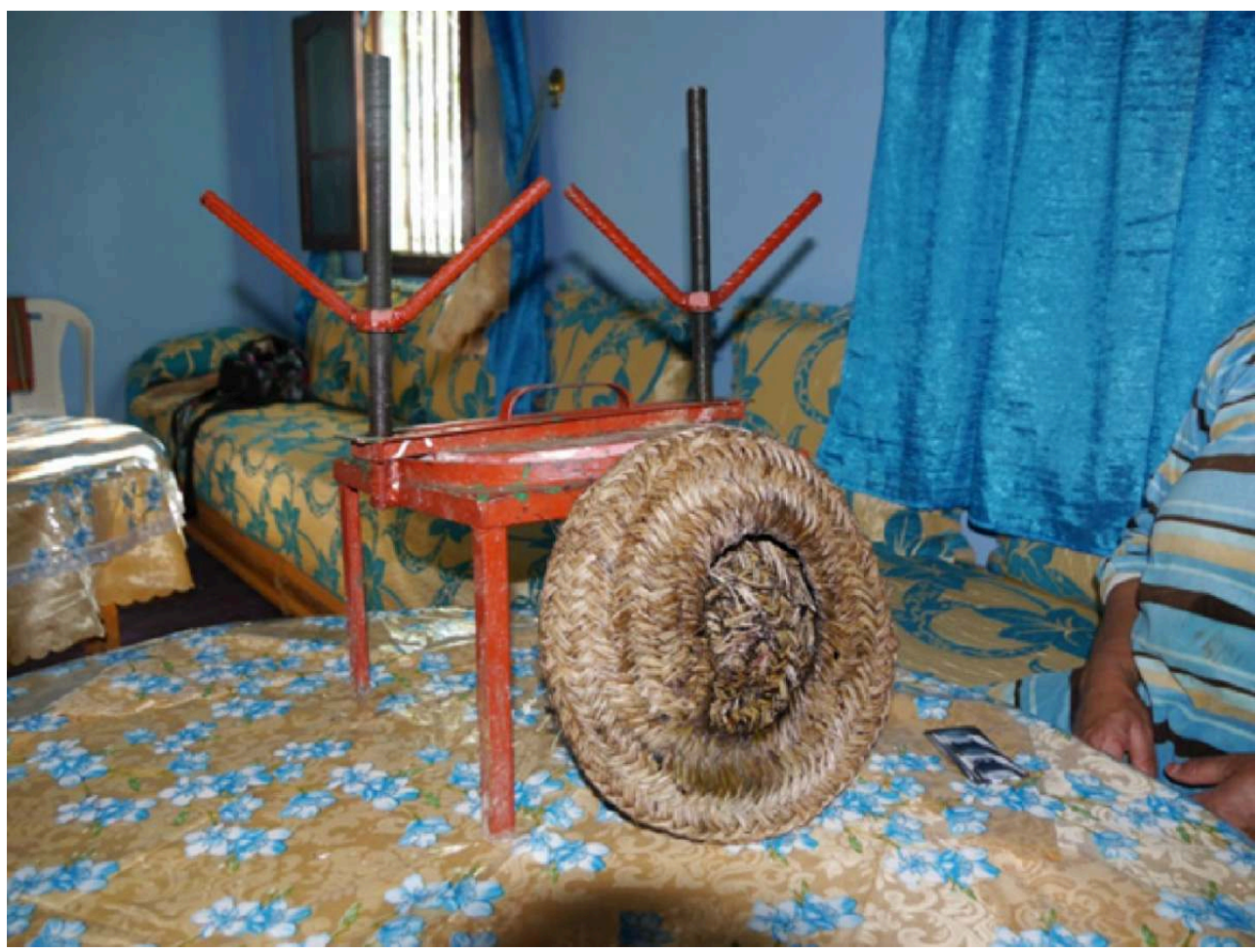

(C) Y. Aumeeruddy-Thomas 2014

44 Nous avons pu observer des presses domestiques en métal également à Bni Ahmed. Gregori Lazarev a acheté une presse initialement en bois blanc sur un souk de Ghafsaï en 1967 auprès d'un vendeur qui proposait plusieurs presses de ce type, et qu'il a teinté ultérieurement, (Figures 4 et 5). À nord Taza, Eəlwāna est aussi connu et couramment utilisé. El Alaoui (2007), a observé une presse en bois à Taounate et rapporte qu'elle était fabriquée à Ain Mediouna et que les scourtins seraient vendus à Taza. 
Figure 4 : Pressoir à huile à double vis latérales en bois, Ghafsaï, nord du Maroc, 1967

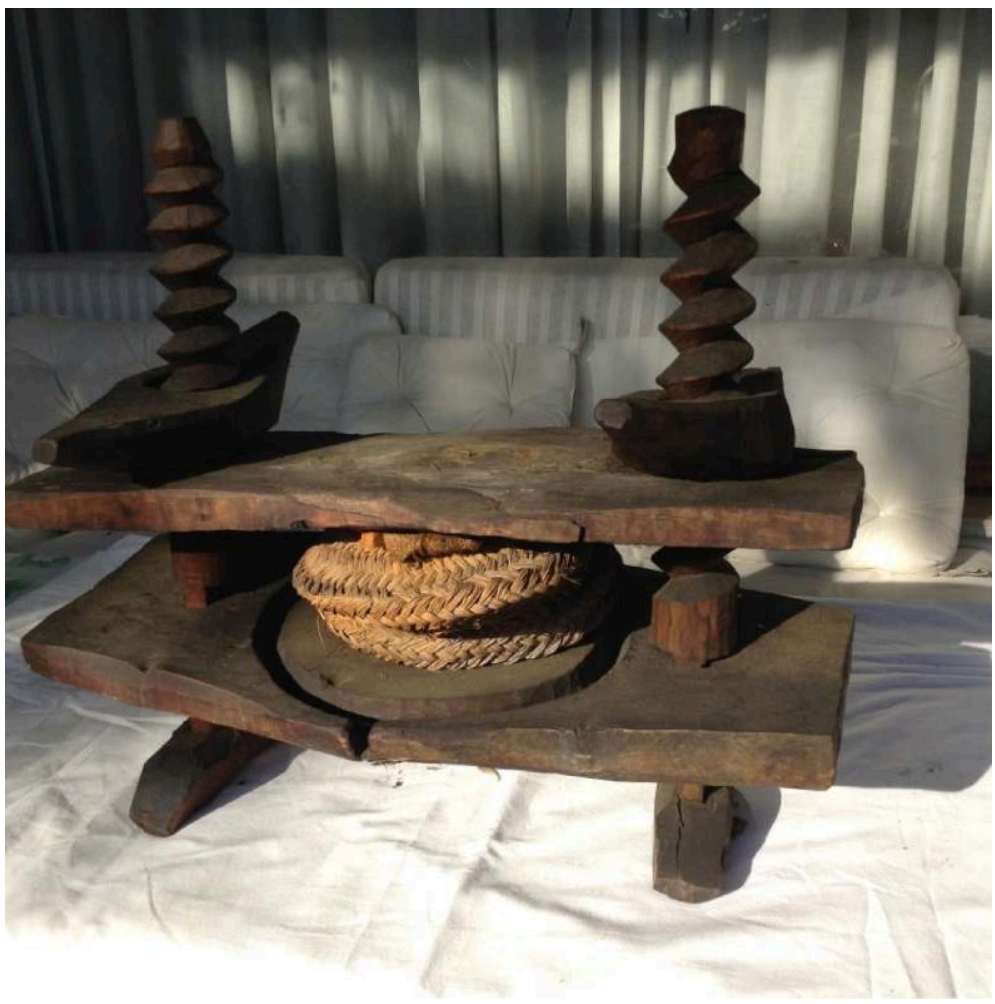

(c) G. Lazarev

Figure 5 : Scourtin, Ghafsaï, nord du Maroc 1967

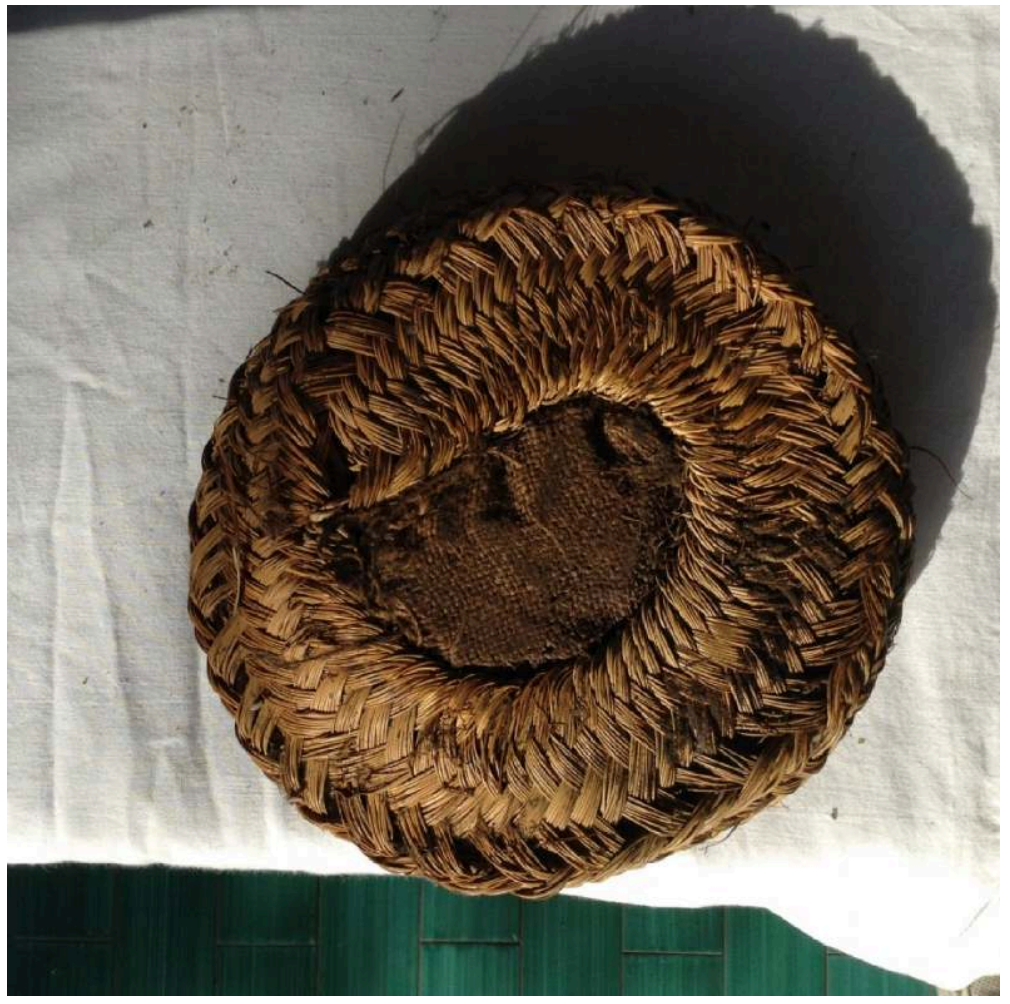

(c) G. Lazarev 
Une fois les olives sorties du four, après y avoir passé une nuit entière dans le forna ${ }^{5}$ (Figure 6) préchauffé et fermé, comme pour le pain, elles peuvent être gardées selon notre hôte toute l'année. Ainsi disent ces femmes, nous pouvons fabriquer cette huile quand nous le souhaitons, sans aller au grand moulin, où l'ensemble des autres olives sont écrasées, malaxées et pressées.

Figure 6 : Le four à Aouina Melha, Ain Mediouna, nord du Maroc

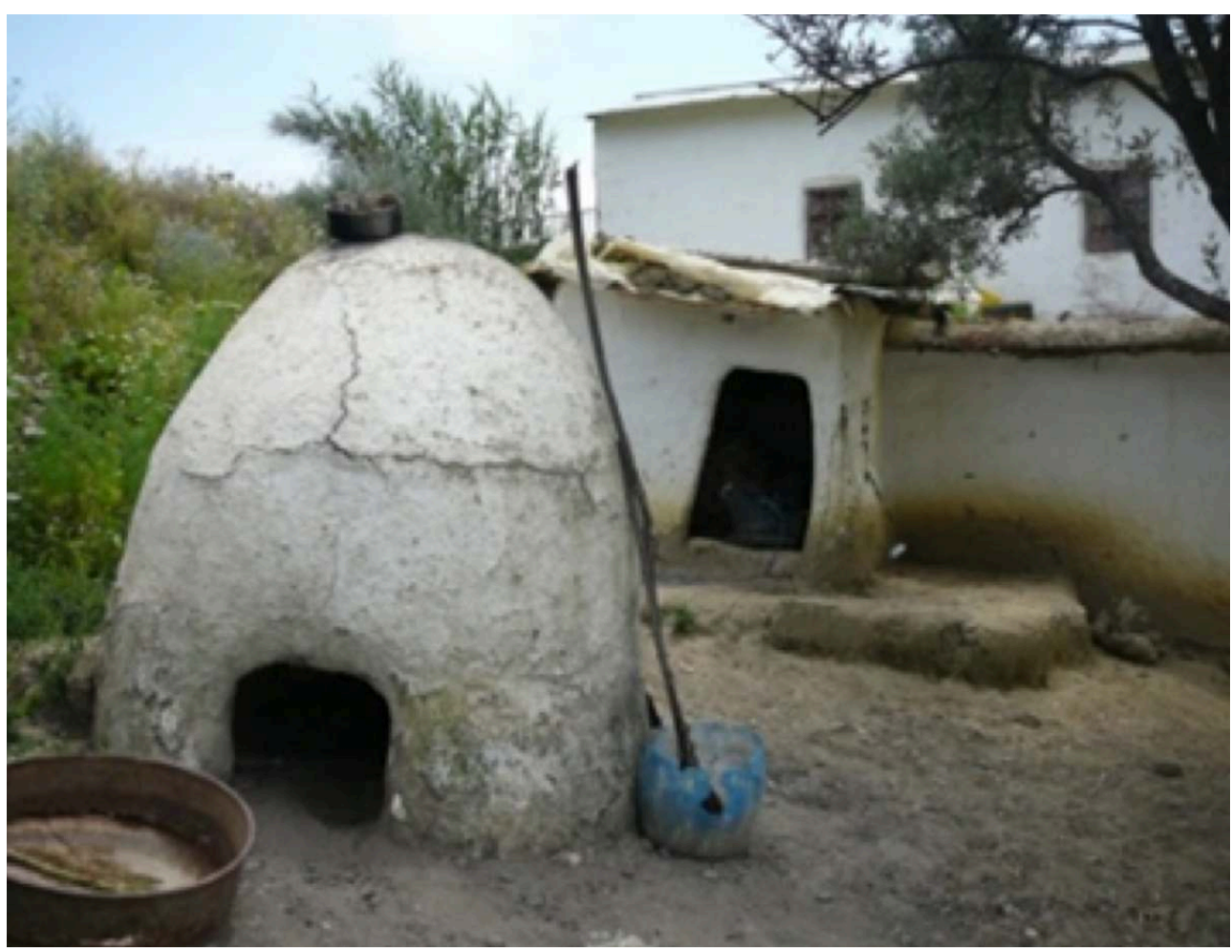

CY. Aumeeruddy-Thomas 2014 
Figure 7 : Les olives confites sorties du four, Aouina Melha, nord du Maroc

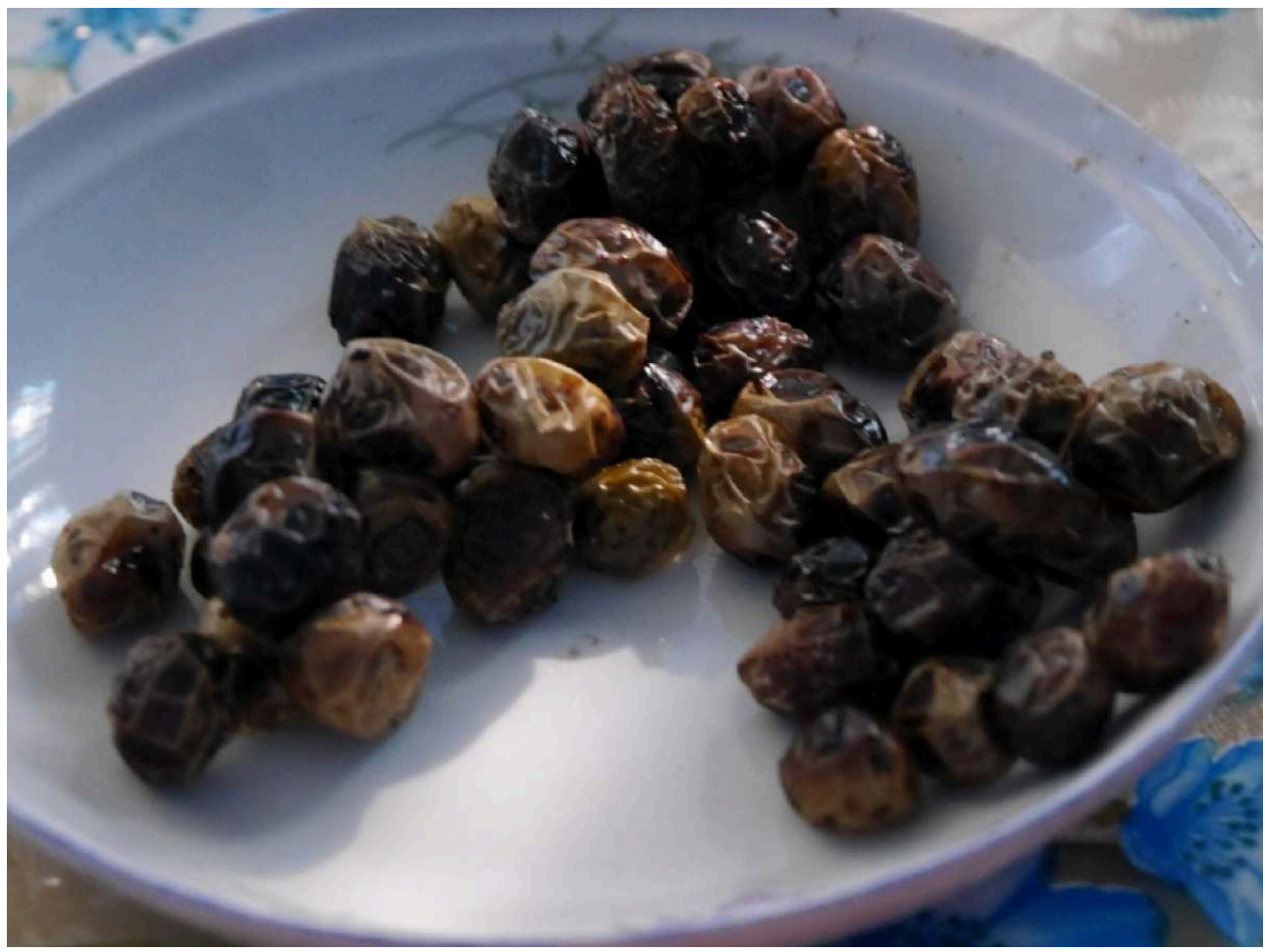

(C) Y. Aumeeruddy-Thomas 2014

Les olives ainsi obtenues sont en quelque sorte confites, avec une couleur légèrement brune, qui donne sa couleur particulière et son parfum à l'huile produite (Figure 8) 
Figure 8 : Huile d'عə/wāna brune et huile d'olive (au premier plan), crêpes (mlāwi) et amandes proposés à Aouina Melha, lors d'un goûter, Ain Mediouna, nord du Maroc

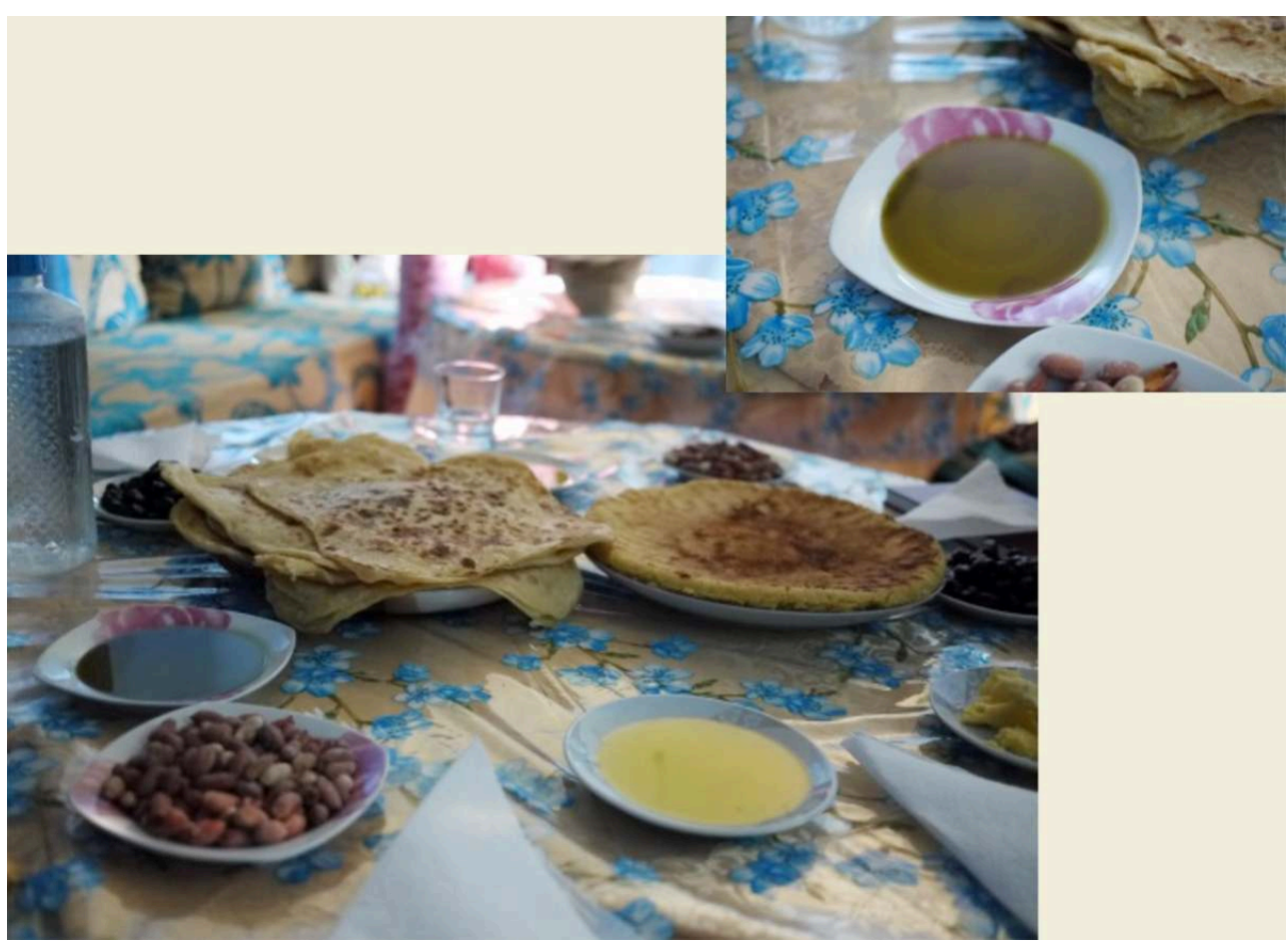

(c) Y. Aumeeruddy-Thomas 2014

\section{À Swetiyyin chez les Hyayna}

Nous nous sommes rendues à Swetiyyin (șwịtiyyīn) en partant d'Ain Mediouna avec deux femmes de Ain Mediouna, pour retrouver la belle-sœur de l'une d'elle (de Aouina Melha) mariée en pays Hyayna. Chez ces derniers, on nous explique que la technique de fabrication d'colwāna y est aussi connue. Cependant il ne nous a pas été indiqué qui des Hyayna (Bédouins) ou des Jbala possédaient cette technique avant l'autre En effet la principale conversation du jour entre les femmes Jbala qui nous accompagnaient et notre hôte Hyayna s'est focalisée rapidement sur différent types de jnūn, entités invisibles présents dans leur vies respectives, un élément fort instructif des modes de vie locaux comme le démontre Araceli González Vázquez (ce volume). Les autres éléments recueillis sont que les habitants de Swetiyyin échangent depuis longtemps au sein des souks tournants (Figure 9), comprenant Ait Aicha, Ain Mediouna, Taounate, Bni Adel, Bni Oulid etc. 
Figure 9 : Extrait de la carte Michelin avec les lieux d'échanges sur des marchés (Taounate, Ain Mediouna, Ain Aicha, Swetiyyin près de Ras el oued, Bouadel, Bni Ouled) et autres sites où la technique $\varepsilon ə / w a ̄ n a$ a été identifiée

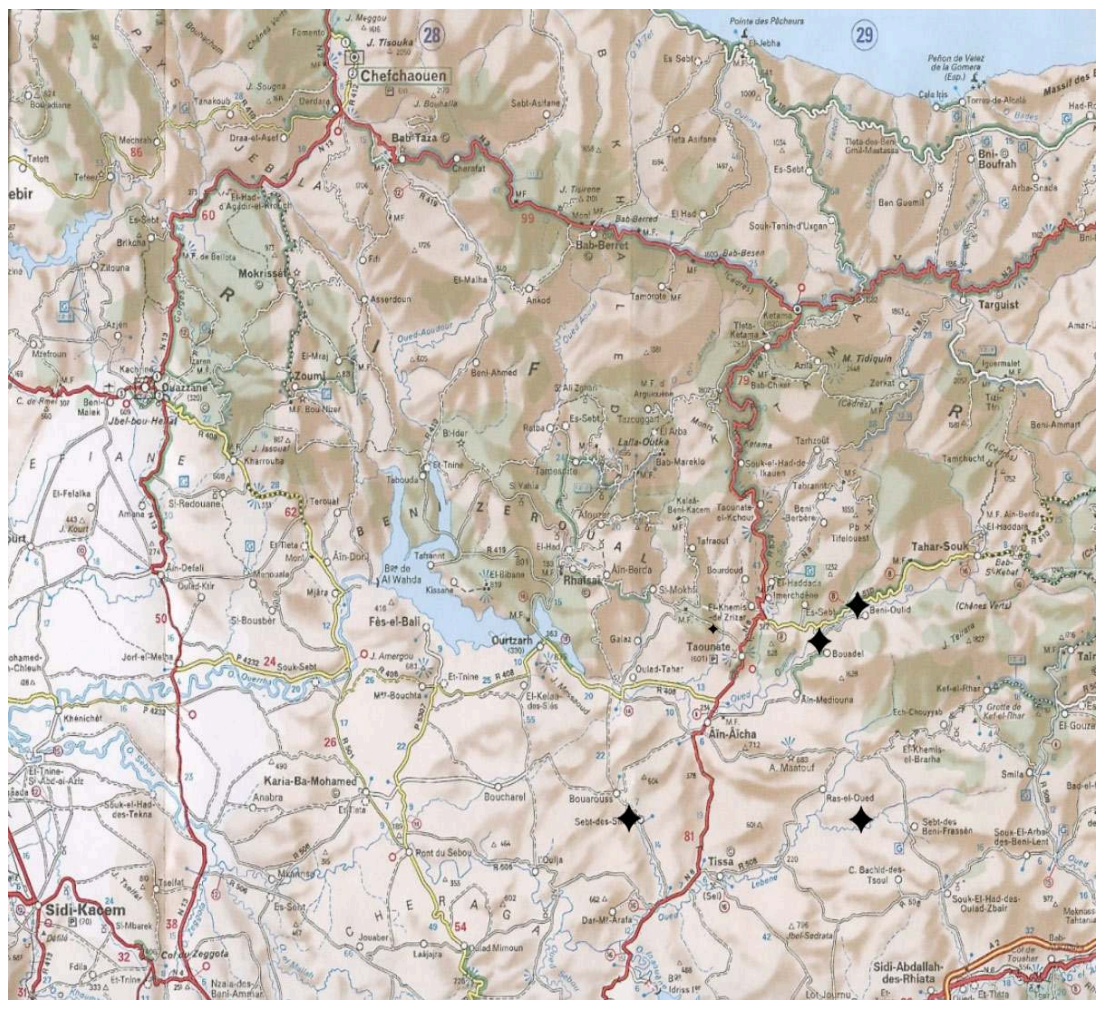

C'est la complémentarité agricole entre ces différentes régions, qui fabriquent ces liens d'échange au sein des marchés. Les Hyayna sont renommés pour leurs céréales, les pailles d'engrain (petit épeautre, Monococcum triticum) ou sqäliya, très utilisés chez eux pour le chaumes des toits, mais aussi très recherchés ailleurs pour les bâts des ânes. Les habitants d'Ain Mediouna vendent des légumes, de l'huile et Bouadel et Bni Oulid des raisins et des figues. Les souks sont de hauts lieux où se croisent les membres de différentes tribus ou confédération tribales et où les parents, hommes et femmes procèdent à de tractations matrimoniales (Troin 1975). Une enquête anthropologique sur ces échanges entre Sweteyyin et Ain Mediouna nous a révélé les échanges matrimoniaux montrés sur la figure 10 (Figure 10). 
Figure 10 : Échanges matrimoniaux entre tribus bédouine Ḥyayna à Swetiyyin et Jbala (Senhaja) et Ain Mediouna
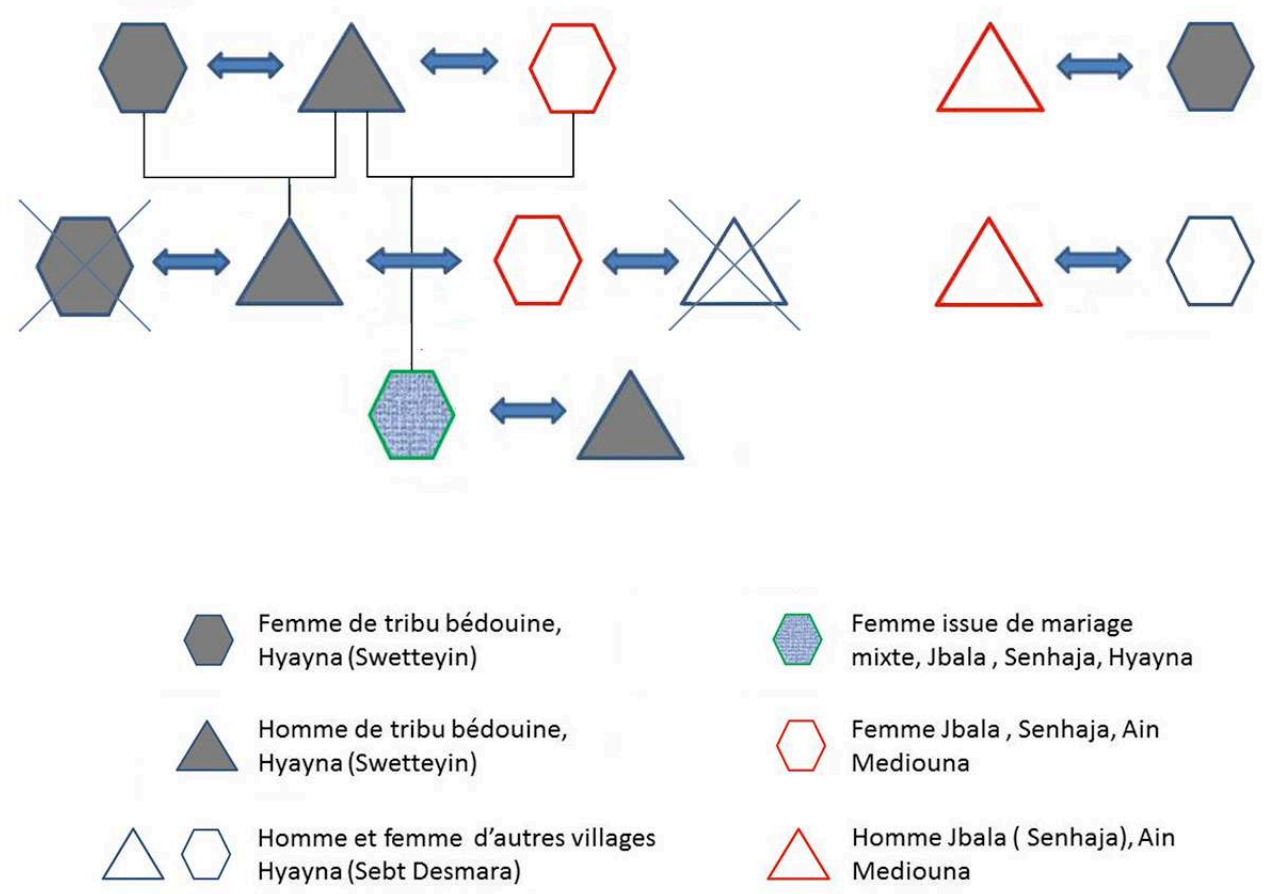

Ce schéma montre qu'à la génération précédente des habitants que nous avons rencontrés, un homme perdant sa femme chez les Hyayna, se marie avec une femme Jbala. Selon notre enquête, cette alliance est fondée sur les relations des hommes de ces deux villages au Souk, qu'ils fréquentent communément depuis au moins deux générations. En échange, un homme jbala se marie avec la sœur de cet homme hyayna. À la génération suivante leur fille se marie avec un homme hyayna, mais le fils issu du premier mariage, ayant aussi perdu sa femme (Hyayna), se marie avec une femme jbala, elle-même veuve. Celle-ci était mariée à un homme décédé d'un autre village hyyayna. Ce schéma très simple qui n'inclut pas tous les échanges matrimoniaux à des générations supérieures et de la génération suivante, montre cependant des échanges matrimoniaux facilités semble-t-il par la situation des veuves et veufs dans deux tribus distinctes. Cette situation rend possible des échanges malgré leur forte distinction culturelle autant au niveau des parlers que des modes de vie. Sachant qu'il s'agit d'une société patrilocale, à savoir que ce sont les femmes qui partent chez leur époux, il est assez facile de comprendre que ce sont les femmes qui favorisent les échanges des techniques alimentaires utilisées uniquement par celles-ci. Ainsi cette technique de fabrication d'huile a pu être échangée à la faveur de ces mariages. Lors de notre séjour à Swetiyyin, notre hôte nous fait à ce titre remarquer que c'est sa femme qui a fabriqué ses ruches, car ce sont des ruches en argile et bale (trāb u tbon) que les Hyayna ne savent pas fabriquer (Figure 11). Les Jbala pour leur part la connaissent très bien notamment pour la fabrication de grands contenants de la même fabrique nommé tonna (Aumeeruddy-Thomas et al. 2014). 
Figure 11 : Rucher observé à Swetiyyin d'une fabrique mettant en œuvre un mélange d'argile et de bale, et un système de montage en colombins propre au pays Jbala

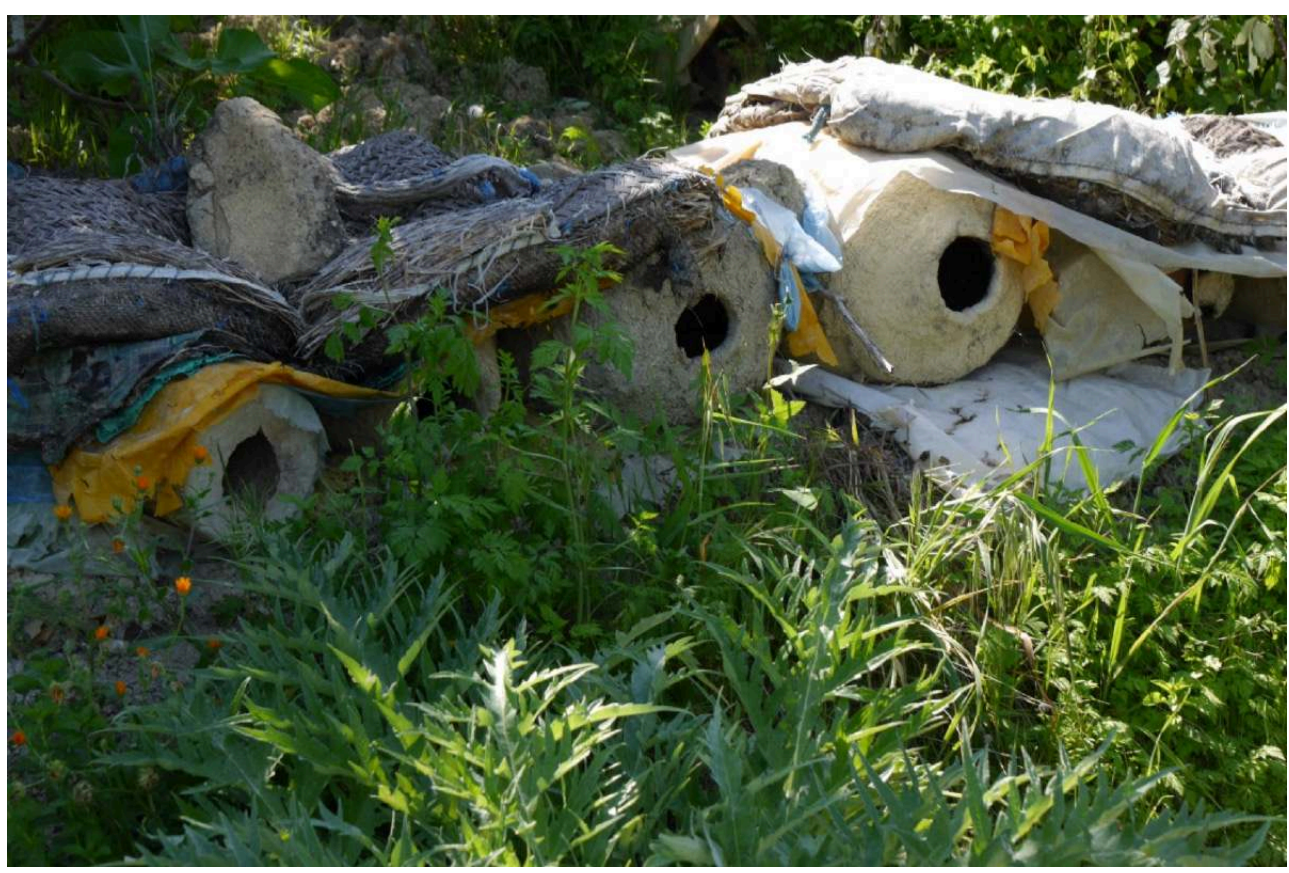

À Ouezzane, nous n'avons recueilli aucune trace à ce jour de Eəlwāna au cours d'une succession d'enquêtes menées en Mai 2009 (Ouezzane et Sidi Redouane, Mai 2013 (Ain Dorij, Zawiya Suffla) et Mars 2016 (Ouezzane, Ain Dorij, et Zawiya Suffla). Les habitants de cette région connaissent l'existence de cette huile mais une dizaine d'interlocuteurs interrogés ont évoqué le fait que Eəlwāna est utilisée dans la région de Taounate ou Taza, mais pas chez eux. Ceci nous amène à considérer que cette technique pourrait être typiquement Jbala, les zones situées le plus au sud où nous l'avons recueillie étant la région des Hyayna, voisine de Ain Mediouna.

\section{t-təryāq : huile vieillie d'olive médicinale}

51 Plusieurs habitants d'Ouezzane nous disent utiliser comme huile médicinale, une huile d'olive vieillie, portant le nom de zit $t$-toryāq. Cette huile est vieillie dans des bouteilles soigneusement fermées et mises à l'abri de la lumière, ou dans des fûts en plastique, possiblement dans des jarres en argile autrefois (nous ne l'avons pas vu). Elle peut être conservée jusqu'à 30-40 ans et possiblement plus. Son acidité augmente au fur et à mesure qu'elle vieillit. Quelqu'un nous dit qu'une goutte sur la main peut créer une plaie; c'est possiblement un effet d'exagération du discours pour éviter des accidents. Cette huile serait selon les Ouezzani utilisée en externe pour traiter les hémorroïdes, mais aussi certaines piqûres de serpent ou de scorpion. Nous retrouvons l'usage de zit ttaryāq à Ain Mediouna, Ain Dorij, Bni Ahmed, Bab Berred, et chez les Hyayna. Araceli González Vázquez (communication personnelle, Juin 2017), en a recueilli l'usage à Fej el-Hanout, fraction Bni Sultane, commune rurale Ghzawa, où l'huile vieillie peut selon ses travaux avoir jusqu'à 40 ans. Selon elle, cette huile est utilisée dans le cadre de certaines pratiques de sorcellerie et ou d'empoisonnement de nourriture nommée tūkal où elle agirait comme un antidote et pour se guérir. Elle est consommée journellement (nous n'avons pas de données sur le degré de dilution!) par la personne qui a été 
empoisonnée. Il semblerait cependant que cette huile serait difficile à trouver de nos jours.

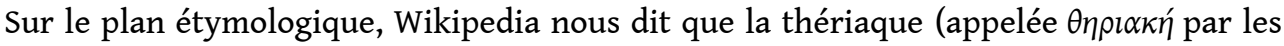
Grecs) est un célèbre contrepoison rapporté à Rome par Pompée, puis complété par Andromaque, médecin de Néron. Sur la rive nord, cette préparation fait appel à des mélanges complexes associant un grand nombre d'ingrédients et a toujours un usage de contrepoison ou parfois de panacée, c'est-à-dire guérissant toutes les maladies. Federico Corriente (Communication personnelle, Saragosse 2015), lors d'un colloque tenu à Saragosse, confirme que ce mot est bien un emprunt du Grec. Dans la composition des thériaques fabriquées par exemple en France à Montpellier, ou en Italie à Venise, on ne trouve jamais d'huile d'olive, ni aucune autre huile. L'usage de la thériaque sous la forme d'un ingrédient unique, l'huile d'olive par les Jbala et les Hyayna, reste donc un mystère quant à l'origine et la diffusion de cet usage.

\section{zīt d-əl-bərri: l'huile d'oléastre}

Pour saisir l'importance de l'huile d'oléastre dans le Rif, il faut s'en éloigner. Ainsi, nous avons systématiquement lors de missions menées à Rabat, Meknès, Marrakech ou dans le Souss, demandé si l'huile d'oléastre se fabriquait localement. Nous avons eue quasiment toujours, la même réponse. Non, mais au Maroc les spécialistes de l'huile d'oléastre, ce sont les Rifains. À Marrakech, l'oléastre est connu sous le nom de zəəbbūž et dans les régions berbères, l'oléastre est le plus généralement nommé azəmmur. De façon générale cette huile est perçue dans le Sud comme une huile amère. Laoust (1983) mentionne l'usage de cette huile amère comme huile de lampe dans le haut Atlas. Dans les jardins de la Menara où nous avons enquêté auprès d'un jardinier en 2006, il a évoqué le fait que le jardin comprenait quelques individus de zəobūž et qu'ils servaient à améliorer la pollinisation des variétés présentes.

54 En pays Jbala, dans le Rif (au sens large, zone traversée par la chaîne rifaine) la perception locale de cette huile se trouve être tout à fait à l'opposé de la vision rencontrée au Sud. Tous les interlocuteurs questionnés, enfants, femmes et hommes, jeunes et vieux, nous disent que l'huile d'oléastre est ḥlūwa, c'est-à-dire douce et l'arbre se nomme l-barri. Deux types d'huile sont fréquemment évoqués zit al-bərri məslāl et $l$ bərri rqīq (litt. étroit, petit). Somme toute, l'huile d'oléastre est à notre connaissance la seule huile au Maroc qui porte un nom de variété. En effet l'huile d'olive normale, ne porte jamais de nom de la variété qui la constitue. La variété rqiq a un fruit allongé et maigre donnant selon les paysans une très bonne huile alors que la variété məslāl est ronde et plus grosse, est également utilisée pour faire de l'huile l-bərri məslāl bien que considérée moins bonne.

Sur le plan linguistique, nous avons interrogé les chercheurs du Groupe de Recherche Jbala lors d'une enquête épistolaire, avec une question posée par Y. A-Thomas concernant la possible signification du terme məslāl ou aməslāl, en 2012 et nous avons eu plusieurs réponses que nous donnons ici afin que le lecteur puisse avoir les diverses interprétations envisageables :

Selon Salem Chaker ${ }^{6}$, en berbère, il existe un terme qualificatif, générique, (a)šemlal / (a)šamlal (avec réalisations locales diverses: rifain ašemrar... ), qui est un dérivé expressif (à préfixe š-) de la racine MLL, «blanc»; le sens de base de (a)šemlal est: 
"blanchâtre, grisâtre ", mais il peut localement (en kabyle par exemple) effectivement désigner des variétés d'oliviers (à feuille particulièrement blanchâtres/grisâtres ).

Selon Mohamed El Mansour, l'olivier connu sous le nom de məslāla est un genre à part, celui qui produit des olives longues, avec peu de teneur en huile, et qu'on enlève à la main (soll, verbe arabe qui veut dire «enlever»), et qu'on utilise comme conserve, à manger, et non pour produire de l'huile. Le nom de məslāla pourrait être traduit comme « celle qu'on enlève à la main ».

58 G. S. Colin, dans son dictionnaire (Iraqui-Sinaceur 1993 (4): 844) donne le sens de « qui engendre beaucoup " pour l'adjectif məslāl.

Ainsi ces différentes interprétations de l'origine de məslāl se recoupent, et semblent bien désigner une grosse olive plutôt de couleur claire, possiblement contenant peu d'huile. Ce terme semble être utilisé comme déterminant ou adjectif associé à l-bərri, pré-figurant un processus de domestication car désignant un type de produits sélectionné sur un arbre non cultivé. Il sert en effet à différencier deux types d'oléastres poussant spontanément, selon le critère de quantité et de qualité de l'huile que donnent leurs fruits, une étape permettant au paysan de sélectionner les arbres avant de décider d'en faire une variété.

Ces données linguistiques concernant maslāl corroborent nos données de terrain, à savoir que les bərri məslāl sont plus gros de même que les variétés d'olive aussi appelées məslāl qui sont aussi plus grosses, et plus précoces, gorgées d'eau avec une couleur vert claire.

61 Les paysans disent que l'huile d'olive est constituée de toutes les variétés qu'ils possèdent et ils y ajoutent l-bərri s'ils en ont un peu. L'huile d'olive se compose d'un mélange, sachant que la grande majorité du verger marocain est constituée d'une variété dominante qui est nommée Picholine marocaine et qui est couramment appelée zaytūn boldi, (Khadari et Moukhli 2016). Le baldi étant toujours défini par rapport à son opposé, zaytūn romi, nous avons pu remarquer que même dans les cas où il s'agit de la même variété Picholine marocaine, la terminologie baldi, s'applique uniquement sur les oliviers reproduits par des techniques de reproduction locale. En effet les Picholines marocaines obtenues des pépinières et traitées dans un contexte industriel, sont des zaytūn romi selon les paysans. Dans la vie courante on dit simplement zaytūn, car il s'agit de la variété archétypique la plus répandue au Maroc. Selon différents interlocuteurs, $l$ bərri rqiq se rapproche des caractéristiques de la Picholine marocaine (zaytūn baldi) qui a une forme longue et allongée, et qui s'oppose dans certains terroirs à une variété grosse et ronde, particulièrement dans le Nord du Maroc, qui se nomme aussi məslāl. (Aumeeruddy-Thomas et al. 2017)

62 En contrepartie, si un paysan a une récolte importante de l-bərri, il peut décider de fabriquer une huile séparée, car au fond il pense qu'elle est meilleure ou est potentiellement meilleure pour la santé. Elle peut se vendre et est alors plus chère. Les enfants qui veulent gagner un peu d'argent les cueillent dans les cours de mosquées et les vendent sur le marché. Dans certains endroits comme à Ain Mediouna, les habitants disent qu'elle est trop difficile à fabriquer. C'est trop long. Ailleurs on nous indique qu'elle fige à faible température ce que certaines personnes valorisent et d'autres considèrent gênant.

63 Les lieux où l'on trouve l-bərri sont très caractéristiques. Tout d'abord c'est un arbre de la forêt que l'on préserve quand on nettoie la forêt pour acquérir une nouvelle terre 
agricole. Les vergers-forêts ainsi obtenus sont des vergers à oliviers greffés sur oléastres comme expliqué plus haut... Au sein de ces vergers il est fréquent de trouver des oléastres non-greffés préservés pour leurs fruits. Nous avons enquêté chez les Masmouda de la tribu des Béni Mesguilda, qui tous systématiquement gardent des individus de l-bərri. été écrasées dans le système traditionnel comprenant une meule en pierre actionnée par un bras latéral nommé rḥa et généralement entraînée par un animal. Une fois cette pâte d'olive obtenue, les femmes y font des creux à la surface en appuyant sur la pâte. L'huile venant se mettre dans ce creux est prélevé est appelée localement l'huile vierge, la meilleure, celle en quelque sorte qui ne subit pas de manipulations outre le passage des olives au moulin. Elle a une valeur inestimable et symbolique et n'est jamais vendue. est encore pratiquée à Ouezzane et dans les régions alentours comme chez les Masmouda. Pour obtenir cette huile, les olives sont écrasées dans des moulins à meule en pierre traditionnels nommés rḥa jusqu'à l'obtention d'une pâte d'olive qui est ensuite mise dans des bassins nommés səhriž et foulée aux pieds par des femmes. Ces bassins étaient traditionnellement fabriqués avec un mortier à base d'argile et de bale, remplacé aujourd'hui par un mortier incluant du ciment. Aucune presse n'est utilisée les femmes traditionnellement foulent avec leurs pieds la pâte d'olive mélangée à l'eau afin de faciliter l'extraction de l'huile. L'huile ainsi extraite est laissée à décanter et surnage sur l'eau. À Bni Ahmed, il nous a été dit que c'était l'huile la plus pure que l'on puisse obtenir, car la décantation sans passage par des systèmes mécaniques de presse ou plus complexes des machines modernes, permet à toutes les impuretés de tomber au 
fond. L'huile ainsi obtenue est récupérée par un tissu nommé sințìt que les femmes passent à la surface pour récupérer l'huile

71 zìt šāmiyya - zit makina. Il s'agit de l'huile obtenue suite au pressage dans des scourtins nommés šāmiyya, des paniers fabriqués en doum ou en jonc, et qui sont remplis de la pâte d'olive et sont empilés et placés dans une presse mécanique, la plus courante étant la presse à double vis latérale fixe. Cette huile a plusieurs caractéristiques selon les habitants: un gout typique qui proviendrait des matériaux végétaux des scourtins; pour faciliter le malaxage, la pâte est additionnée d'eau froide et non d'eau chaude; elle est obtenue par le passage très lent de la meule entraînée par un animal. L'ajout d'eau exclusivement froide donnerait une qualité à cette huile qui est qualifiée de lourde ou épaisse, avec plusieurs termes qui sont xātra ou tqïla. Ces caractéristiques sont valorisées aux yeux des Jbala en plus de sa couleur caractéristique jaune-vert et son opacité, par opposition à des huiles qualifiées de zìt makina qui sont qualifiées de xfifa (légères) et žārya (qui court). Ces caractéristiques sont aussi valorisées ailleurs au Maroc comme nous l'avons observé dans l'arrière-pays de Marrakech. Concernant zìt makina, il s'agit de celle extraite par différentes machines qui ont été importées au cours de l'histoire. Les Jbala pensent que la pression est trop forte et l'eau additionnée trop chaude, ce qui dénaturerait la qualité de l'huile. Cependant ils perçoivent évidemment l'intérêt de ces autres formes de production, dans la mesure où ça va vite et que cela se vend bien, non pas localement mais sur des circuits plus longs. Il n'en reste pas moins qu'ils préfèrent garder pour leurs usages personnels les huiles plus artisanales.

Le tableau 1 rassemble les différentes terminologies et labels concernant les huiles d'olive et d'oléastre du Rif et du Pré-Rif

Tableau 1 : Terme relatifs aux moulins et aux huiles recueillis dans le Nord du Maroc

\begin{tabular}{|c|c|c|}
\hline El Alaoui (2007) & Aumeeruddy-Thomas \& Caubet et autres références analysées & Traductions et notes techniques \\
\hline $\begin{array}{l}\text { chamya/chamma/chwami, } \\
\text { Taounate, Ouezzane }\end{array}$ & šămiyya & Scourtin : paniers en doum ou en jonc \\
\hline fitur/lfitor & zît l-fitör & $\begin{array}{l}\text { Tourteaux (résidus secs de la pâte d'olive) aux usages variés : } \\
\text { combustion (Moulieras 1985), fabrication du savon noir, matériau de } \\
\text { construction. }\end{array}$ \\
\hline frna & $\begin{array}{l}\text { forna (Ain Mediouna, Bni Ahmed, Ouezzane) } \\
\text { forrān. (Msek) }\end{array}$ & $\begin{array}{l}\text { Four en ogive construit par les femmes (cuisson du pain, } \\
\text { déshydratation des olives. } \\
\text { Ouverture à hauteur des bras à Msek ou au ras du sol à } \\
\text { Ain Mediouna et Ouezzane }\end{array}$ \\
\hline gs'a & $\begin{array}{l}\text { gəșæa (Ain Mediouna, Msek) } \\
\text { Gaca' (selon Moulieras 1899, cité par Monkachi 1997) }\end{array}$ & $\begin{array}{l}\text { Grand plat en argile rouge ; à fond plat utilisé pour pétrir du pain ou } \\
\text { pour recueillir et malaxer l'huile }\end{array}$ \\
\hline jfna & tažefnit (Msek) & Idem \\
\hline Im'șriya & meīșa (Ain Mediouna) & $\begin{array}{l}\text { Petite presse à huile à deux vis, à usage domestique, mobile } \\
\text { pouvant s'échanger entre maisons proches }\end{array}$ \\
\hline $\begin{array}{c}\text { Alwana } \\
\text { (Taounate) }\end{array}$ & \begin{tabular}{|l|} 
Ealwāna [Ain Mediouna, Bni Ahmed, Bab Taza, Bab Berret, \\
Chaouen, Msek (Rif Oriental), Sweteyyin (Hyayna, bédouins)
\end{tabular} & $\begin{array}{l}\text { Huile des prémices obtenues en début de saison avec des olives } \\
\text { immatures grillées au four }\end{array}$ \\
\hline $\begin{array}{c}\text { rha } \\
\text { (Nord du Maroc) }\end{array}$ & rhạa, tous le nord dans toutes les régions Jbala & $\begin{array}{l}\text { Moulin à bras avec une meule de pierre, fixe parfois situé dans un } \\
\text { moulin ou au sein de l'oliveraie en plein air (Ouezzane, Mesmouda, } \\
\text { Bni Ahmed etc.) }\end{array}$ \\
\hline Zit Ihayla/zit tryak & $\begin{array}{l}\text { zīt t-taryāq ( Jbala : Ain Mediouna, Ain Dorij, Bni Ahmed, Bab } \\
\text { Berred, Ghzawa, Hyayna, Sweteyyin) }\end{array}$ & Huile d'olive vieillie, antidote, et autres usages médicinaux \\
\hline Ssahrij (Ouezzane, Zoumi) & sehriž (Ouezzane, Mesmouda) & Bassin de foulage des olives \\
\hline Sntafa & sentit & Etoffe de laine pour récupérer l'huile dans les bassins de décantation \\
\hline \multirow[t]{7}{*}{ Zit Tahlwant (Souss) } & & $\begin{array}{l}\text { Olives vertes cueillies à la main sont brisées entre deux pierres puis } \\
\text { mises à cuire à la vapeur dans un couscoussier et pressées entre } \\
\text { les doigts. }\end{array}$ \\
\hline & derra d əl-hhāyāti, (Msek) & Carré de tissu en cotonnade fine \\
\hline & zīt d-ol-ma (Ouezzane, Mesmouda) & $\begin{array}{l}\text { Huile foulée aux pieds à Ouezzane et Mesmouda. Aurait existé } \\
\text { anciennement à Bni Ahmed. Moulieras }\end{array}$ \\
\hline & zît d-el-berri meslāl, (Ouezzane, Mesmouda) & Huile d'oléastre \\
\hline & zīt l-bərri rqīq (Ouezzane, Mesmouda) & Huile d'oléastre \\
\hline & zît makina & Huile obtenue de machines modernes \\
\hline & zīt zarūsa & $\begin{array}{l}\text { Huile vierge obtenu par prélèvement directe au moulin, avant toute } \\
\text { forme de pressurage ou de décantation. C'est I'huile qui surnage à la } \\
\text { surface de la pâte d'olive après le malaxage par la meule. }\end{array}$ \\
\hline $\begin{array}{l}\text { Zet mn el Botem (Catineau, } \\
\text { cité par El Alaoui) }\end{array}$ & zït d-dro (Msek) & $\begin{array}{l}\text { Huile de pistachier térébinthe } \\
\text { A noter que Batam est le nom donné au Nord du Maroc au Pistachier } \\
\text { de l'atlantique (Pistachia atlantica) }\end{array}$ \\
\hline
\end{tabular}




\section{Conclusion}

Ce papier met en évidente des savoirs et des savoir-faire des paysans Jbala contribuant à maintenir un large dispositif d'extraction d'huiles d'olive ainsi que des labels locaux, socialement reconnus et partagés. Ceci indique un ancien savoir-faire qui a pu se développer par différentes influences, possiblement romaines, mais aussi par le biais de l'inventivité des paysans et des modalités de diffusion à travers le Rif, au-delà des frontières linguistiques locales. Ce papier montre des similitudes en terme de savoirs autour des huiles entre les Jbala, la communauté arabophone rifaine de Msek et les Hyayna (Bédouins).Le caractère contemporain de ces savoirs et savoir-faire montre de surcroît le fait qu'ils s'inscrivent dans le cadre d'une culture alimentaire où l'huile d'olive apparait comme un indicateur très fort d'appartenance au pays (bəldi), de lien à des dimensions sacrés (bāraka), et de typicités des huiles associées à des techniques connues et perpétués, différenciant les hommes et les femmes.

Ealwāna apparaît à l'évidence comme une huile à usage domestique, exclusivement développée par les femmes. Celles-ci ont probablement participé à sa diffusion par le biais d'échanges matrimoniaux entre les Jbala et des groupes frontaliers linguistiquement et culturellement distincts, notamment les Hyayna, groupes d'origine bédouine sédentarisé au XVI ${ }^{\mathrm{e}}$ siècle. Compte tenu de la densité de lieux en pays Jbala utilisant Ealwāna, nous pouvons avancer l'idée que ce sont bien les Jbala qui ont transmis cette technique aux Hyayna (Bédouins) arrivés seulement au $\mathrm{xV}^{\mathrm{e}}$ sur ces territoires.

La technique recueillie à Msek, utilisant un tissu darra el hāāāti, bien que relevant d'un geste différent de celui observé à Ain Mediouna, rappelle l'usage de tissus, sințit par les femmes à Ouezzane pour le zit d al-ma. La technique simple de concassage des olives pour Ealwāna sur une meule constituée d'une roche plate légèrement creuse rappelle la meule néolithique, recensée par de nombreux archéologues en Méditerranée, un système dès lors très archaïque, que pourtant les femmes ne semblent pas considérer comme tel. Celle-ci est en effet une des plus anciennes meules utilisée en Méditerranée basée sur l'usage d'une pierre légèrement creuse et une deuxième pierre utilisée en va et vient ou pour concasser (http://www.archeobase.be/page_meules_neo.html). Bien que nous ne puissions pas dater exactement ce procédé, il témoigne d'une ancienne relation à la fabrication d'huile. L'intérêt des femmes à préserver cette technique est liée au fait qu'elle procure une grande indépendance par rapport au moulin généralement contrôlé par des hommes ; elle est fondée sur leur propres récoltes selon des gestes bien différents de ceux des hommes et mettant en jeu des liens entre femmes proches partageant le même moulin. Elles valorisent aussi le fait qu'il s'agit d'une période de l'année où les stocks d'huile de l'année précédente peuvent être épuisés, ainsi que la possibilité de stocker les olives grillées pour produire des petites quantités d'huile selon leurs besoins. Enfin elles montrent généralement un grand intérêt pour la diversité en soi des types d'huiles, Ealwāna apportant selon elles une note et un goût très spécial. El Alaoui (2007) propose l'idée que la diffusion de cette technique de presse mobile ait pu diffuser par le biais de l'imprimerie développée à Fès, ce qui est possible concernant cette presse mais pas en ce qui concerne toute la chaîne opératoire permettant de produire Eəlwāna. Nos travaux suggèrent en effet que des savoir-faire paysans et en particulier des femmes, très probablement précédant l'invention de l'imprimerie, favorisés par des échanges au sein des souks et la circulation des femmes 
lors de mariages intra ou inter tribaux, ont pu constituer un moteur majeur de diffusion de cette technique, plus que l'idée d'une diffusion par l'écriture et les travaux d'érudits développés dans des villes.

Le foulage aux pieds des olives, nous semble également dater d'une période ancienne et avait été également noté par Mouliéras (1895). Or plusieurs femmes évoquent le fait qu'elles aiment produire de cette façon et elles aiment cette huile. C'est là également une question d'attachement et un lien fort entre femmes qui ne peut s'expliquer autrement que par le plaisir de produire ensemble.

zìt d-əl-ma et zit Earūsa sont aussi exclusivement destinées à des usages domestiques. Toutes ces huiles néanmoins font partie de réseaux de dons pour les proches. Partir avec une bouteille d'huile de cette qualité, c'est emmener aussi avec soi la bāraka, qui s'est imprégnée à l'huile tout au long de son processus de fabrication. Cette notion de bāraka, associée à la bénédiction divine est également liée au traitement de l'arbre, à la nature des porte-greffes qui poussent spontanément, à la récolte qui ne dépend pas uniquement de la volonté des hommes. En effet dans le Rif, l'abondance des récoltes est souvent une affaire de bāraka.

Bien que nous n'ayons pas parlé des moulins traditionnels, nous pourrions dire brièvement que lorsqu'on apporte sa récolte au moulin, chaque famille surveille son huile, l'ordre de passage compte et elle est dépendante de la relation au propriétaire du moulin. Après l'obtention de l'huile, divers rituels peuvent marquer la fin de ce processus, comme le sacrifice d'un poulet, le partage de nourriture ensemble, des chants et des youyous. L'ensemble de cette chaîne opératoire n'est donc pas que production d'huile mais aussi production de culture, de liens sociaux, d'identité et de maintien d'équilibres avec l'Autre, les éléments intangibles de la nature comme nous l'explique en détail Araceli González Vázquez (ce volume) concernant les pratiques alimentaires autour de l'hospitalité. Ces ensembles de pratiques ne sont plus présentes dans des moulins modernes, ce qui laisse à penser que ces lieux ne sont plus des lieux de liens sociaux ou des lieux de culture. En effet, bien que certains de ces moulins assurent aux propriétaires que leur huile est bien celle provenant de leurs propres olives, il s'agit d'une habitude qui se perd. Dans le cas où le paysan vende simplement ses olives au moulin au poids et récupère en retour un certain nombre de litres, la relation entre la production, les soins apportés, de la production à la récolte est rompue.

79 Alors que l'huile d'oléastre peut se vendre - et se vend cher -, elle est aussi conservée pour un usage domestique car elle est perçue comme étant très bonne au goût mais aussi pour la santé. La très grande originalité de ces huiles, est que leur différenciation en des termes distinguant des huiles à l'échelle variétale, en soi montre les prémices classificatoires d'une domestication variétale. En effet on pourrait penser que le fait de différencier les deux types d'huile de l-barri pourrait guider a posteriori le choix de l'une ou l'autre des deux variétés d'oléastre pour ensuite les sélectionner et en faire des variétés cultivées (greffées). Cette donnée vient compléter nos travaux sur la domestication de l'olivier dans le Nord du Maroc (Aumeeruddy-Thomas et al. 2017). Ceci met en exergue la très grande importance des savoirs des paysans Jbala, et réaffirme comme le propose Chaker (2013) que le monde berbère, dans notre cas arabisé au $\mathrm{VII}^{\mathrm{e}}$ siècle pour les groupes jbala, ait pu jouer un rôle considérable dans le développement des connaissances sur les olives. 
80 Le t-taryāq est médicinal et constitue un antidote comme expliqué plus haut. Cette huile vieillie peut être reliée par sa terminologie aux Grecs, et pourrait constituer une trace lointaine des échanges entre les Grecs et le monde berbère. La thériaque des rives Nord est cependant infiniment plus complexe que celle utilisée au Maroc et rien sur le plan de leur préparation ne les rapproche.

81 Les huiles traditionnelles de moulin sont pour leur part vendues sur des circuits courts dans des marchés locaux, marchés tournants qui facilitent les échanges sociaux.

82 Enfin, toutes ces huiles participent à cet acte hautement social qui est de manger ensemble. Outre la valeur nutritive indéniable de ces huiles, elles sont pour les plus courantes le plus souvent présentes aux trois repas de la journée du petit déjeuner au soir, et parfois lors de petites collations. Pourquoi les habitants du Rif et particulièrement les Jbala ont-ils gardé une telle diversité de types d'huiles. Selon El Alaoui (2003) ce serait l'attrait pour un goût du produit du pays, ce que nous confirmons. En outre, nous pensons qu'il s'agit d'un faisceau très vaste de raisons car ces huiles semblent en effet se concentrer dans les régions Jbala du Pré-Rif, du Rif central et jusqu'aux communautés arabophones situées à la frontière de la berbérophonie, dans toutes les zones où les vergers-forêts d'oliviers greffées sur oléastres sont les plus répandus. La diversité de ces huiles, il nous semble, pourrait être fortement reliée à l'ensemble des savoirs associés à ces vergers-forêts du Pré-Rif, du Rif Central et l'ancienneté des techniques qui perpétuent ces formations agro-sylvopastorales qui produisent en outre bien d'autres produits tels que les plantes adventices comestibles (voir Clochey et Aumeeruddy-Thomas, ce volume). Cette maîtrise historique de la production peut expliquer le soin équivalent apporté à la production de cette grande diversité d'huile, et le fort intérêt dans le produit final et sa multiplicité de formes et de goûts.

83 Ces vergers-forêts en même temps que les modalités de productions des huiles font cependant face à des projets de développement du Plan Maroc Vert qui consistent précisément à les remplacer par des savoirs autres, avec des oliviers venus d'ailleurs et reproduits à grande échelle en vue de produire une huile aux qualités prévues pour l'exportation. Les modalités de production des huiles suivent dès lors une chaîne opératoire qui implique des critères de qualité qui suivent celles des huiles européennes ou internationales. Les soutiens à la production poussent les agriculteurs à intégrer ces nouvelles pratiques, y compris des engagements dans différentes coopératives. Ces évolutions nous amènent à réfléchir au rôle et à la responsabilité des chercheurs dans la nécessité de mettre en exergue dans un premier temps la valeur hautement patrimoniale non seulement de ces huiles, mais aussi des agroécosystèmes qui les produisent. Sans doute un engagement entre chercheurs et acteurs de ces territoires serait important à envisager afin de préserver certains sites à forte valeur patrimoniale.

84 Sur le plan linguistique, nous avons un riche vocabulaire autour de l'olive qui exprime aussi cette richesse de savoirs et de savoir-faire ainsi que des perceptions organoleptiques très spécifiques de ces différentes huiles. Un projet plus vaste afin de compléter ce lexique nous paraît désormais primordial, car relevant d'une culture menacée. 
Carte du positionnement des Hyayna dans le Pré-Rif, Maroc selon El Moubaraki

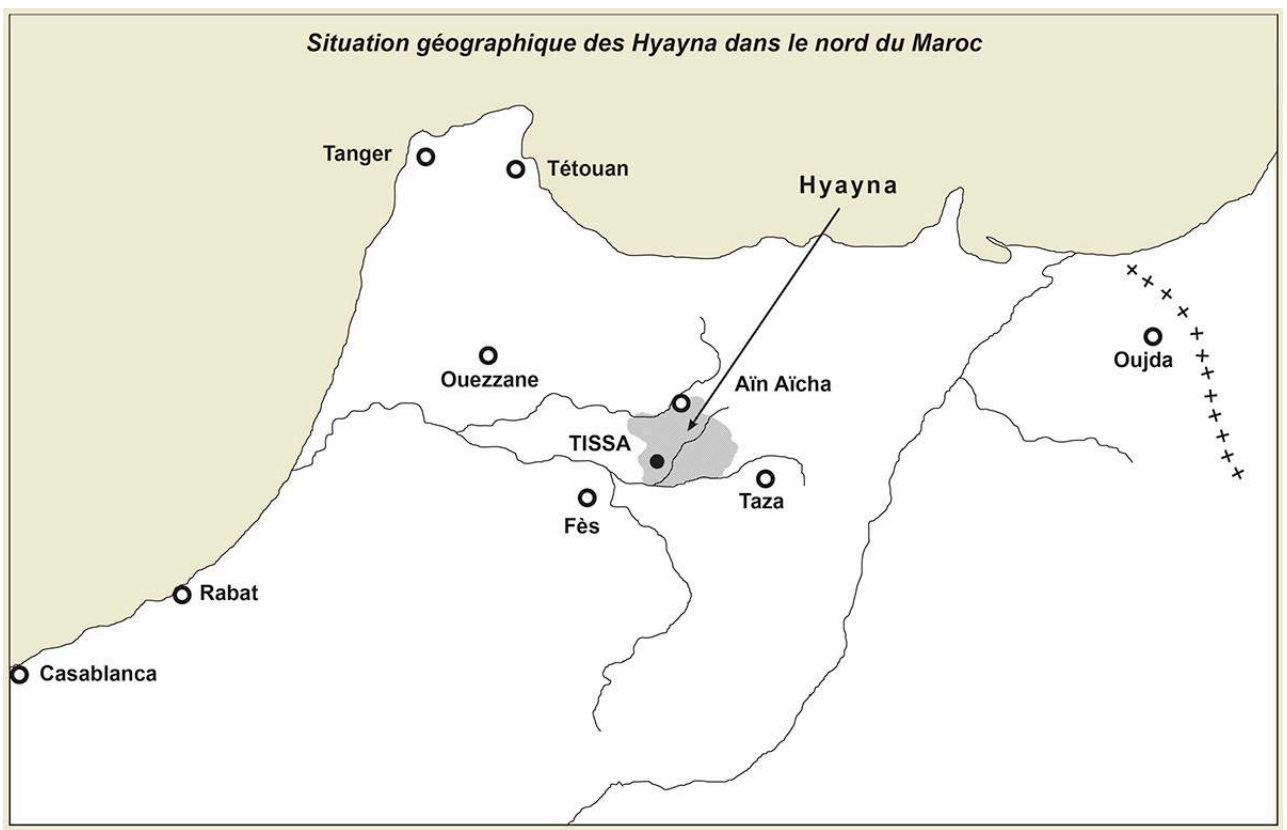

\section{BIBLIOGRAPHIE}

Amouretti M.C. 1986 - Le pain et l'huile dans la Grèce antique: de l'araire au moulin. Paris, Les Belles Lettres, Paris.

Aumeeruddy-Thomas Y., Caubet D., El Ghazaz F. \& Clochey L. 2014 - Céréales et huiles d'olive: savoirs, savoir-faire techniques et relations d'échanges entre deux groupes sociaux voisins, Jbala (Aïn Mediouna) et Hyayna (Switeyyin) du Nord du Maroc. Congreso internacional. Lenguas, memorias y lecturas de la alteridad:Jbala, Ghomara y Rif(Norte de Marruecos), Saragosse 8-9-10 de septiembre de 2014.

Aumeerudddy-Thomas Y., Bailly A., Alleaume S. \& Hmimsa Y. 2016 - Grafted oleaster-olive agrosylvopastoral systems in Northern Morocco. In Thiébault S. et J-P. Moatti, (Eds) The Mediterranean Region Under Climate Change. A Scientific Update. , Marseille , IRD Editions: 523-532. http://fr.calameo.com/read/00331938471

Aumeeruddy-Thomas Y., Moukhli A., Haouane H., Khadari B. 2017 - Ongoing domestication and diversification in grafted olive-oleaster agroecosystems in Northern Morocco. Regional Environmental Change. 17:1315-1328 DOI 10.1007/s10113-017-1143-3

Ballouche A \& Marinval P2003 - Données palynologiques et carpologiques sur la domestication et l'agriculture dans le Néolithique ancien du Maroc Septentrional (Site de Kaf Taht el Ghar). Revue d'Archéométrie, 49-54 [En ligne] doi : 10.3406/arsci.2003.1041

Besnard G. Khadari B. , Navascués M., Fernández-Mazuecos M., Arrigo N. , Baali-Cherif D., Brunini-Bronzini de Caraffa V , Santoni S., Vargas P., Savolainen V. 2013 - The complex history of the olive tree: from Late Quaternary diversification of Mediterranean lineages to primary 
domestication in the northern Levant, Proceedings of the Royal Society B,-Biological Sciences 280: 1756 DOI: $10.1098 / \mathrm{rspb} .2012 .2833$

Brun J-P. 1986 - L'oléiculture antique en Provence. Les huileries du Département du Var. Revue archéologique de Narbonaise, Supplément 15, Paris, Edition du CNRS,..

Barazani O, Westberg E., Hanin N., Dag A. , Kerem Z, Tugendhaft Y, Hmidat M., Hijawi T, Kadereit JW 2014 - A comparative analysis of genetic variation in rootstocks and scions of old olive trees, a window into the history of olive cultivation practices and past genetic variation. BMC Plant Biology: 14-146, 14:146 DOI: 10.1186/1471-2229-

Bellakhdar J. 2006 - Plantes médicinales au Maghreb et sons de base. Précis de phytothérapie moderne Casablanca, Editions Fennec, $386 \mathrm{p}$.

Camps G. 1961 - Massinissa, ou les débuts de l'histoire, 1961, Alger, Imprimerie officielle.

Camps G. 1985 - « Pour une lecture naïve d'Hérodote. Les récits libyens (IV, 168-199) », Storia della Storografia, $7: 38-59$.

Chaker S. 2013 - D’où vient l'olivier ? « Des barbares ont-ils enseigné aux Hellènes ? » Enquête à propos du nom de l'olivier, grec ou berbère, Connaissance Hellénique 3 Commentaires, http:// ch.hypotheses.org/601

Chandezon C. 2007 - L'olivier dans le monde grec ancien. Quelques aspects des recherches récentes. In Dumond L. Durand S. (Ed) L'olivier dans l'Europe méditerranéenne de l'Antiquité à nos jours. Terroir, Paysage et Economie. LIAME, Bulletin du Centre d'Histoire de l'art des époques modernes et contemporaine de l'Europe méditerranéenne et de ses périphéries. Presse Universitaire de la Méditerranée, Montpellier : 13-42.

Charafi, J., El Meziane, A., Moukhli , A., Boulouha, B., El Modafar, C., Khadari, B. 2008 - Menara gardens : a Moroccan olive germplasm collection identified by a SSR locus-based genetic study, Genetic Resources and Crop Evolution: 893-900. https://doi.org/10.1007/s10722-007-9294-6

Diez C.M., Trujillo I., Barrio E., Belaj A., Barranco D., Rallo L 2011 - Centennial olive trees as a reservoir of genetic diversity. Annals of Botany 108: 797-807. DOI: 10.1093/aob/mcr194

Diez CM, Trujillo I., Martinez-Urdiroz N., Barranco D., Rallo L., Marfil P., S Gaut B. 2015 - Olive domestication and diversification in the Mediterranean Basin. New Phytologist 206: 436-447 URL : http://tc.revues.org/4541; DOI : 10.4000/ tc.4541

El Alaoui N. 2007 - Une presse à huile au Maroc Techniques \& Culture: 48-49 [en ligne] URL : http://tc.revues.org/4541; DOI : 10.4000/tc.4541

El Faiz M. \& Bendaoud R 2000 - Jardins de Marrakech. Arles, Actes Sud, 186pEl Jettari K. 2017 Contact de parlers arabe et berbère en zone du Rif : Région de Bni Itteft. In : Vicente Á., Caubet D. \& Naciri Azzouz A. (éds.), La région du Nord-Ouest marocain: Parlers etșpratiques sociales et culturelles. Zaragoza, Prensas de la Universidad de Zaragoza : 315-326.

El Jettari K. 2017 - Contact de parlers arabe et berbère en zone du Rif : Région de Bni Itteft. In : Vicente Á., Caubet D. \& Naciri Azzouz A. (éds.), La région du Nord-Ouest marocain: Parlers et pratiques sociales et culturelles. Zaragoza, Prensas de la Universidad de Zaragoza : 315-326.

El Moubaraki M. 1989 - Marocains du Nord. Entre la mémoire et le projet. Paris, Ciemi l'Harmatan, $251 \mathrm{p}$.

Guernier E. 1947 - L'encyclopédie coloniale et Maritime, Tunisie, 6e MILLE , Paris .Edition Encyclopédie de l'Empire Français: 222- 223 
Hmimsa Y. 2009 - L'agrodiversité de l'agroécosystème à l'arbre : cas du Rif (Nord du Maroc). Thèse de doctorat. Tétouan, Université Abdelmalek Essaadi - Faculté des Sciences, $328 \mathrm{p}$ Iraqui Sinaceur Z. (Dir.) 1993 - Le dictionnaire Colin d'arabe dialectal marocain (arabe français). Rabat : Al Manahil, 2107 p. (8 volumes).

Frank T 1926 - A Commentary on the Inscription from Henchir Mettich in Africa. The American Journal of Philology, 47(2), 153-170. doi:10.2307/290009

Kaniewski D., Van Campo E., Boiy T., Terral JF., Khadari B., Besnard B. 2012 - Primary domestication and early uses of the emblematic olive tree: palaeobotanical, historical and molecular evidence from the Middle East, Biological Reviews, pp. 000-000. doi: 10.1111/j. 1469-185X.2012.00229.X

Khadari B. \& Moukhli A. 2016 - Peut-on parler de l'olivier au Maroc sans la variété « Zitoun Beldi » ou «Picholine marocaine ». In Ater M. , Essalouh L. , Ilbert H. , Moukhli A. , Khadari B (Eds.) . L'oléiculture au Maroc de la préhistoire à nos jours : pratiques, diversité, adaptation, usages, commerce et politiques. Montpellier, CIHEAM : 67-78 (Options Méditerranéennes : Série A. Séminaires Méditerranéens; n. 118)

Kislev M E, Nadel D, Carmi I (1992) Epipalaeolithic (19,000 B. P.) cereal and fruit diet at Ohalo II, Sea of Galilee, Israel. Review of Paleobotany and Palynology 73: 161-166.

Laoust E. 1983 - (2ième édition) Mots et choses berbères. Notes de linguistiques et d'ethnographie. Dialectes du Maroc, Paris Ed Augustins Challamel, 531p

Mediterra 2012 - The Mediterranean Diet for Sustainable Regional Development/ International Centre for Advanced Mediterranean Agronomic Studies (CIHEAM). - Paris: Presses de Sciences Po,

Maghdad A. 1993 - El habla árabe en el Aduar de Msek (Textos, Traducciones, Notas y Compendio). Mémoire de licence d'espagnol non publié sous la direction de Simon Lévy, Rabat, Université Mohamed V, 1992-1993, 88 p.

Meillet A. 1975 - Linguistique historique et linguistique générale,. A propos du nom du vin et de l'huile. Paris, Champion : 297-304.

Monkachi M. 1997 - L'alimentation traditionnelle dans les campagnes du nord du Maroc. In : Médiévales. Cultures et nourriture de l'occident musulman. Essais dédiés à Bernard Rosenberg (33) : 91-102

Mouliéras A. 1895 - Le Maroc inconnu - 2ème partie : exploration du Djebala (Maroc Septentrional). Paris, Augustin Challamel, 931 p.

Ramon-Münoz R. 2007 - Concurrence et mutation dans le marché international de l'huile d'olive, 1850 -1938, In Dumond L. \& Durand S. (Ed.) L'olivier dans l'Europe méditerranéenne de l'Antiquité à nos jours. Terroir, Paysage et Economie. LIAME, Bulletin du Centre d'Histoire de l'art des époques modernes et contemporaine de l'Europe méditerranéenne et de ses périphéries. Presse Universitaire de la Méditerranée, Montpellier : 216-245.

Salavert A. 2008 - Olive cultivation and oil production in Palestine during the early Bronze Age (3500-2000 b.c.): The case of Tel Yarmouth, Israel. Vegetation History and Archaeobotany, 17(SUPPL. 1), 53-61. https://doi.org/10.1007/s00334-008-0185-3

Szymanski E 1970 - Les tribus de « Guich » et le Makhzen sous le règne de Sidi Mohammed Ben Abd Allah. Revue de l'Occident musulman et de la Méditerranée (8) 195-202 http:// www.persee.fr/doc/remmm_0035-1474_1970_hos_8_1_1044 
Terral JF, \&A rnold-Simard G 1996 - Beginnings of Olive Cultivation in Eastern Spain in Relation to Holocene Bioclimatic Changes. Quaternary Research 46: 176-185.

Toutain MJ. (1902) - L'Inscription de Henchir Mettich. Un nouveau document sur la propriété agricole dans l'Afrique Romaine. Académie des Inscriptions et Belles Lettres. 11 (1) : 31-82 http:// www.persee.fr/doc/mesav_0398-3587_1902_num_11_1_1081

Troin JF. 1975 - Les souks marocains: marchés ruraux et organisation de l'espace dans la moitié nord du Maroc, Aix-en-Provence : Edisud, I : 502p ; II : 28pl.

Zohary D., M Hopf M., Weiss E 2012 - Domestication of plants in the old world, Oxford University Press, Oxford. 1406 (1581) 175 DOI: 10.1126/science.187.4174.319

\section{ANNEXES}

\section{Annexe 1}

\section{NOTES}

1. Concernant les modes de transcription des termes arabes, voir Aumeeruddy-Thomas et al. ce numéro

2. Les transcriptions sont de Mouliéras.

3. gașEa, selon notre transcription de ce terme aussi recueilli à Msek et Ain Mediouna.

4. Nous les avons longuement enregistrés lors de notre mission de mai 2014 à Switiyyin, mais la description de leur parler, qui est très différent de celui d'Aouina Melha et qui présente des traits bédouins, reste à faire car ceci représente une situation inhabituelle dans le Rif (Pré-Rif). L'origine bédouine des Hyayna est cependant attestée (voir El Moubaraki 1989, Szymanski 1970)

5. À Aouina Melha, le four se dit forna, alors qu'à Msek (voir Caubet et Aumeeruddy-Thomas, ce volume), on l'appelle farrān.

6. Salem Chaker est le Directeur de l'Encyclopédie berbère, Professeur de Berbère, Université d'Aix-Marseille.

\section{RÉSUMÉS}

Dans cet article, nous nous intéressons centralement aux produits de l'olivier et de l'oléastre du Nord du Maroc, aux modalités techniques d'obtention de différents types d'huile et ce que ces produits représentent pour les habitants. Il s'agit de comprendre si le Rif détient une particularité sur ce sujet. Nous suggérons que ces techniques et les savoirs et savoir-faire sur les huiles ont pu influencer des processus pré-domesticatoire et de création variétale dans le nord du Maroc. Nous cherchons à comprendre si le Rif a une spécificité à ce sujet. Nous discutons en outre, comment s'effectue des échanges au sein des groupes arabophones du Rif sur ce sujet en particulier entre arabophones Jbala de Ain Mediouna et leurs voisins, des groupes sociaux aux parlers Bédouins, les Hyayna avec qui ils échangent régulièrement. L'approche conjointe ethnobotanique et linguistique, à travers l'analyse de certains termes tels que məslāl utilisé pour 
qualifier un type d'huile d'oléastre zìt d-əl-bərri məslāl, montre en effet une forme binaire de classification de variétés d'huiles d'oléastre, ayant pu influencer la sélection de variétés du même nom répandues notamment dans le nord du Maroc. Une diversité remarquable de types d'huiles portent des appellations locales distinctes : zìt t-toryāq, Eəlwāna, zìt šāmiyya, zìt d-əl-bərri məslāl, zìt al-bərri rqīq, zit d-əl-ma, zìt l-Earūsa, etc. et correspondent à des procédés d'extraction allant des meules et des moulins des plus anciens, de type néolithique aux plus modernes. Cette diversité semble indiquer que les paysans de nord du Maroc possèdent des savoirs et des savoir-faire, anciens et significatifs autour de l'olivier et de l'oléastre, et y compris, en amont, la maîtrise de la technique de greffage sur oléastres. Ces savoirs menacés par l'industrialisation de la production oléicole au Maroc, sont néanmoins d'une valeur patrimoniale, culturelle et économique inestimable pour la sécurité alimentaire locale, et le maintien de processus de diversification variétale, un sujet de grande actualité en matière de développement durable.

In this article, we are centrally interested in the products of the olive tree and oleaster in Northern Morocco and in the technical conditions used to obtain table olives and several types of oils, and in what these products represent for the inhabitants. We aim to understand if the Rif offers any peculiarity. We suggest that these techniques, knowledge and expertise on oils have influenced pre-domesticatory and breeding processes in Northern Morocco. We will also discuss the exchanges within the Arabic-speaking groups of the Rif on this subject, and especially between Ain Mediouna who are Jbala Arabic speakers and their neighbors, groups speaking a Bedouin Arabic dialect, the Hyayna with whom they exchange regularly. The joint ethnobotanic and linguistic joint approach, through the analysis of certain terms such as məslāl used to describe a type of wild olive oil, zitəl bərri məslāl, shows a binary classification of varieties of oils, which could have led to the selection of varieties of the same name spreading mostly in the North of Morocco. A remarkable diversity of types of oils bear different local names: zit t-taryāq, Eəlwāna, zìt šāmiyya, zìt d-əl-bərri məslāl, zìt əl-bərri rqīq, zìt d-əl-ma, zìt Earūsa, etc. They correspond to extraction processes ranging from the oldest millstones and mills dating from the Neolithic, to the most modern. This diversity suggests that farmers in Northern Morocco have old and significant types of knowledge and know-hows about olive trees and the oleaster, including the mastering of the oleaster grafting techniques. This knowledge, threatened by the industrialization of the oil production in Morocco, are however of heritage value -both culturally and economically- and invaluable for local food security, and the maintenance of the varietal diversification process, a highly topical subject for sustainable development.

\section{INDEX}

Keywords : olive, oleaster, extraction techniques, mills, grafting, domestication, varietal diversity, nutrition, linguistics, ethnobotanics

Mots-clés : olive, oléastre, huiles, techniques d'extraction, moulins, greffage, domestication, diversité variétale, alimentation, linguistique, ethnobotanique

\section{AUTEURS}

\section{YILDIZ AUMEERUDDY-THOMAS}

Directeur de Recherche, CNRS, Centre d'Écologie Fonctionnelle et Évolutive (UMR5175 CEFE) Campus CNRS, 1919 route de Mende - 34293 Montpellier cedex 5, France yildiz.aumeeruddy-Thomas@cefe.cnrs.fr 


\section{DOMINIQUE CAUBET}

Professeur émérite d'arabe maghrébin, INALCO. LaCNAD et Centre Jacques Berque - 16 Bd. A. Blanqui - 75013 PARIS

caubet.dominique@yahoo.fr 